\title{
Uncertainty Modeling by Bilattice-Based Squares and Triangles
}

\author{
Chris Cornelis, Ofer Arieli, Glad Deschrijver, and Etienne E. Kerre
}

\begin{abstract}
In this paper, Ginsberg's/Fitting's theory of bilattices, and in particular the associated constructs of bilattice-based squares and triangles, is introduced as an attractive framework for the representation of uncertain and potentially conflicting information, paralleling Goguen's $\mathcal{L}$-fuzzy set theory. We recall some of the advantages of bilattice-based frameworks for handling fuzzy sets and systems, provide the related structures with adequately defined graded versions of the basic logical connectives, and study their properties and relationships.
\end{abstract}

Index Terms-Bilattices, bilattice-based squares and triangles, implicators, MV-algebras, negators, t-norms and t-conorms.

\section{INTRODUCTION}

B ILATTICES are algebraic structures that were introduced by Ginsberg in [1], [2] as a general and uniform framework for a diversity of applications in artificial intelligence. In particular, he treated first-order theories and their consequences, truth maintenance systems, and formalisms for default reasoning. In a series of papers, Fitting then showed that bilattices are very useful tools for providing semantics to logic programs (see, e.g., [3]-[5]), a thesis that was later vindicated in [6]-[8]. Several works have shown that bilattices may serve as a foundation of other areas, such as computational linguistics [9] and distributed knowledge processing [10]. In particular, a family of bilattice-based logics and corresponding proof systems were introduced in [11]-[13], where it was shown that bilattices are useful as the underlying algebraic structures of formalisms for reasoning with imprecise information (see also [14], [15]). This point was recently made explicit in the context of fuzzy set theory, where we have shown (see [16], [17]) that bilattices, and in particular the associated constructs of bilattice-based squares and triangles, provide an elegant framework for bridging between intuitionistic fuzzy sets [18] and interval-valued fuzzy sets [19], [20], two common extensions of fuzzy sets.

The aim of this paper is to substantiate this bilattice-based framework by equiping it with suitable implementations for the common logical connectives of negation, conjunction, disjunction and implication. As is well known from fuzzy set theory, an adequate choice for these operations, inspired by the properties we want them to satisfy, often determines to a great extent the strength of the applications that rely on them.

Manuscript received December 1, 2004; revised August 23, 2005 and August 29,2005 . The work of C. Cornelis was supported by the National Science Foundation-Flanders.

C. Cornelis, G. Deschrijver, and E. E. Kerre are with the Fuzziness and Uncertainty Modelling Research Unit, Department of Applied Mathematics and Computer Science, Ghent University, B-9000 Gent, Belgium (e-mail: Chris.Cornelis@UGent.be; Glad.Deschrijver@UGent.be; Etienne.Kerre@UGent.be).

O. Arieli is with the Department of Computer Science, The Academic College of Tel-Aviv, Tel-Aviv 61161, Israel (e-mail: oarieli@mta.ac.il).

Digital Object Identifier 10.1109/TFUZZ.2006.881444
Fortunately, we do not have to start our investigation from scratch. Instead, it turns out that ideas from both $\mathcal{L}$-fuzzy set theory [21] and bilattice theory [1], [22] can go a very long way in helping us pinpoint the "best" choice for these connectives, allowing for a positive synergy between the contributing theories. Incidentally, the present paper can also be viewed as a generalization to the lattice-valued and bilattice-valued case of previous papers [23]-[26] that refer to particular forms of 'triangle' and 'square', in which the underlying structure is the unit interval.

The rest of the paper is organized as follows: first, in Section II, we recall some elementary concepts of bilattices and bilattice-based squares and triangles. Section III is the heart of this paper, in which we consider proper representations of logical connectives in our framework: the first part (Section III-A) establishes the representation of involutive negators, the second part (Section III-B) explores the idea of $\mathcal{L}$-representability in the definition of $\mathrm{t}$-norms and $\mathrm{t}$-conorms for modeling conjunction and disjunction, and the last part (Section III-C) introduces several ways of representing implication connectives and examines the relationships among them, as well as their relations to other connectives. In particular, the choice of the "right" negator and the existence of an associated MV-algebra are explored. Finally, in Section IV we hint on the application potential of our bilattice-based framework and conclude.

\section{PRELIMINARIES}

In this section, we review some basic definitions and notions that pertain to bilattices in general, and bilattice-based squares and triangles in particular. For other expositions of these structures and the motivations behind them, we refer to [16] and [17].

Definition: A prebilattice [22] is a structure $\mathcal{B}=$ $\left(B, \leq_{t}, \leq_{k}\right)$, such that $B$ is a nonempty set containing at least two elements, and $\left(B, \leq_{t}\right),\left(B, \leq_{k}\right)$ are complete lattices. A bilattice [1] is a structure $\mathcal{B}=\left(B, \leq_{t}, \leq_{k}, \neg\right)$, such that $\left(B, \leq_{t}, \leq_{k}\right)$ is a prebilattice, and $\neg$ (the "negation") is a unary operation on $B$ satisfying, for every $x, y$ in $B$, the following properties:

1) $\neg \neg x=x$;

2) if $x \leq_{t} y$ then $\neg x \geq_{t} \neg y$;

3) if $x \leq_{k} y$ then $\neg x \leq_{k} \neg y$.

In the sequel, following the usual notations for the basic bilattice operations, we shall denote by $\wedge$ (respectively, by $\vee)$ the $\leq_{t}$-meet (the $\leq_{t}$-join) and by $\otimes($ respectively, by $\oplus$ ) the $\leq_{k}$-meet (the $\leq_{k}$-join) of a bilattice $\mathcal{B} . f$ and $t$ denote the $\leq_{t}$-extreme elements, and $\perp, \top$ denote the $\leq_{k}$-extreme elements of $\mathcal{B}$. Intuitively, these elements can be perceived as "false," "true," "unknown" (i.e., neither true nor false) and 
"contradictory" (both true and false), respectively. The two partial orders $\leq_{t}$ and $\leq_{k}$ are taken to represent differences in the degree of truth and in the amount of information (respectively), conveyed by the assertions.

By Definition 1, the negation $\neg$ of a bilattice $\left(B, \leq_{t}, \leq_{k}, \neg\right)$ is an involution with respect to the lattice $\left(B, \leq_{t}\right)$ and an order preserving operation of the lattice $\left(B, \leq_{k}\right)$. Conversely, a conflation, - , is an involution of $\left(B, \leq_{k}\right)$ and an order preserving operation of $\left(B, \leq_{t}\right)$. When a conflation can be defined in a given bilattice, it is usual to require also that both kinds of involution commute: for every $x$ in $B,-\neg x=\neg-x$.

The following proposition recalls some important properties of bilattices.

Proposition 1: Let $\mathcal{B}=\left(B, \leq_{t}, \leq_{k}, \neg\right)$ be a bilattice. Then:

- [1] For every $x, y$ in $B$ :

a) $\neg(x \wedge y)=\neg x \vee \neg y, \neg(x \vee y)=\neg x \wedge \neg y, \neg(x \otimes y)=$ $\neg x \otimes \neg y, \neg(x \oplus y)=\neg x \oplus \neg y$.

b) $\neg f=t, \neg t=f, \neg \perp=\perp, \neg \top=\top$.

- [22] If $\mathcal{B}$ is a bilattice with a conflation -, then for every $x, y$ in $B$ :
a) $-(x \wedge y)=-x \wedge-y,-(x \vee y)=-x \vee-y$, $-(x \otimes y)=-x \oplus-y,-(x \oplus y)=-x \otimes-y$.
b) $-f=f,-t=t,-\perp=\top,-\top=\perp$.

Definition 2: A bilattice $\mathcal{B}=\left(B, \leq_{t}, \leq_{k}, \neg\right)$ is distributive if all (twelve) possible distributive laws concerning $\wedge, \vee, \otimes$, and $\oplus$ hold. A distributive bilattice is called classical if it has a conflation - that commutes with $\neg$, such that $x \vee-\neg x=t$ for every $x$ in $B$.

When $\left(B, \leq_{t}, \leq_{k}, \neg\right)$ is classical, then $(B, \wedge, \vee,-\neg)$ is a Boolean algebra. The reason for considering this conflation-negation combination rather than, e.g., $\neg$ by itself is that, amongst others, $\perp \vee \neg \perp=\perp \neq t$, making $\neg$ an inadequate choice for a Boolean negation (see, also Section III-C, and in particular the paragraph that surrounds Proposition 13). In the sequel we shall sometimes abbreviate the combination $-\neg$ by $\sim$.

Definition 3: Let $\mathcal{L}=\left(L, \leq_{L}\right)$ be a complete lattice. A (bilattice-based) square is a structure $\mathcal{L}^{2}=\left(L \times L, \leq_{t}, \leq_{k}, \neg\right)$, where, for every $\left(x_{1}, x_{2}\right),\left(y_{1}, y_{2}\right)$ in $L^{2}$,

1) $\neg\left(x_{1}, x_{2}\right)=\left(x_{2}, x_{1}\right)$;

2) $\left(x_{1}, x_{2}\right) \leq_{t}\left(y_{1}, y_{2}\right) \Leftrightarrow x_{1} \leq_{L} y_{1}$ and $x_{2} \geq_{L} y_{2}$;

3) $\left(x_{1}, x_{2}\right) \leq_{k}\left(y_{1}, y_{2}\right) \Leftrightarrow x_{1} \leq_{L} y_{1}$ and $x_{2} \leq_{L} y_{2}$.

An element $\left(x_{1}, x_{2}\right)$ of a square $\mathcal{L}^{2}$ may intuitively be understood such that $x_{1}$ represents the amount of belief for some assertion, and $x_{2}$ is the amount of belief against it. This corresponds to Atanassov's idea [18] of distinguishing between a membership component $\mu_{A}(u)$ and a nonmembership component $\nu_{A}(u)$, with the amendment that in the case of a square no restriction like $\mu_{A}(u)+\nu_{A}(u) \leq 1$ for every $u$ in $U$ is imposed. It follows, then, that squares can be regarded as a generalized form of Atanassov's [18] intuitionistic fuzzy sets (see, also [17]).

Example 1: Let $\mathcal{L}_{2}=(\{0,1\}, \leq)$ and $\mathcal{L}_{3}=(\{0,1 / 2,1\}, \leq)$, where $\leq$ in each case is the usual ordering. The corresponding squares $\mathcal{L}_{2}^{2}$ and $\mathcal{L}_{3}^{2}$ are shown in Fig. ${ }^{1}{ }^{1}$ It is easy to verify that both squares are distributive, but only $\mathcal{L}_{2}^{2}$ is classical.

\footnotetext{
${ }^{1}$ These structures are commonly referred to as $\mathcal{F O U R}$ (after Belnap's [27], [28] original four-valued logic) and $\mathcal{N} \mathcal{I} \mathcal{N} \mathcal{E}$ (see, e.g., [12], [13]), respectively.
}

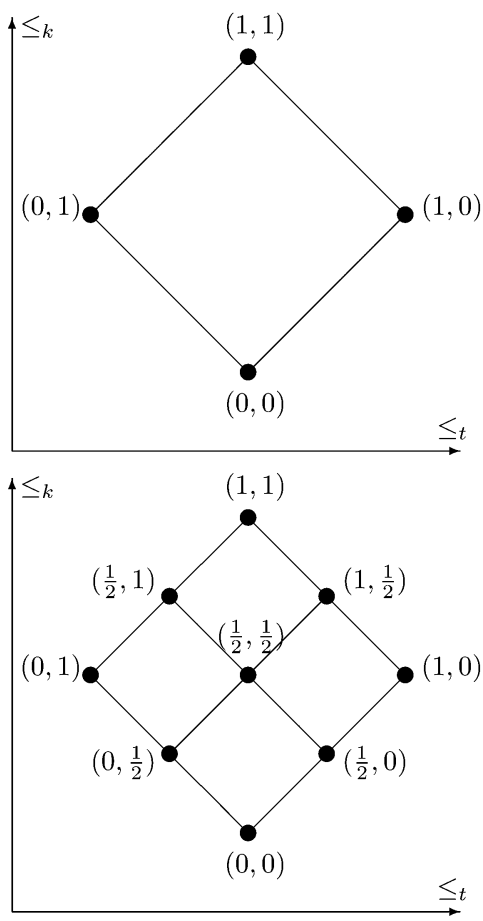

Fig. 1. Squares $\mathcal{L}_{2}^{2}$ and $\mathcal{L}_{3}^{2}$.

Denoting the join and meet operations of a complete lattice $\mathcal{L}$ by $\wedge_{L}$ and $\vee_{L}$, respectively, for every $\left(x_{1}, x_{2}\right),\left(y_{1}, y_{2}\right)$ in $L^{2}$, we have

$$
\begin{aligned}
& \left(x_{1}, x_{2}\right) \wedge\left(y_{1}, y_{2}\right)=\left(x_{1} \wedge_{L} y_{1}, x_{2} \vee_{L} y_{2}\right) \\
& \left(x_{1}, x_{2}\right) \vee\left(y_{1}, y_{2}\right)=\left(x_{1} \vee_{L} y_{1}, x_{2} \wedge_{L} y_{2}\right) \\
& \left(x_{1}, x_{2}\right) \otimes\left(y_{1}, y_{2}\right)=\left(x_{1} \wedge_{L} y_{1}, x_{2} \wedge_{L} y_{2}\right) \\
& \left(x_{1}, x_{2}\right) \oplus\left(y_{1}, y_{2}\right)=\left(x_{1} \vee_{L} y_{1}, x_{2} \vee_{L} y_{2}\right)
\end{aligned}
$$

Moreover, denoting $0_{\mathcal{L}}=\inf L$ and $1_{\mathcal{L}}=\sup L$, it holds that

$$
\begin{aligned}
\perp_{\mathcal{L}^{2}} & =\left(0_{\mathcal{L}}, 0_{\mathcal{L}}\right) \\
\top_{\mathcal{L}^{2}} & =\left(1_{\mathcal{L}}, 1_{\mathcal{L}}\right) \\
t_{\mathcal{L}^{2}} & =\left(1_{\mathcal{L}}, 0_{\mathcal{L}}\right) \\
f_{\mathcal{L}^{2}} & =\left(0_{\mathcal{L}}, 1_{\mathcal{L}}\right)
\end{aligned}
$$

and when $\mathcal{N}$ is an involution of $\mathcal{L}$, then $-\mathcal{N}$ defined for every $\left(x_{1}, x_{2}\right)$ in $L^{2}$ by $-\mathcal{N}\left(x_{1}, x_{2}\right)=\left(\mathcal{N}\left(x_{2}\right), \mathcal{N}\left(x_{1}\right)\right)$, is a conflation of $\mathcal{L}^{2}$.

It is easy to verify that every square $\mathcal{L}^{2}$ is distributive when $\mathcal{L}$ is distributive. Structures of the form $\mathcal{L}^{2}$ and their generalization to the cartesian product of two lattices were studied by Fitting [3], [5], [22] and Avron [29], [30] as a general method for constructing bilattices.

The second bilattice-based structure under investigation here is due to Fitting [22].

Definition 4: Let $\mathcal{L}=\left(L, \leq_{L}\right)$ be a complete lattice, and let $I(L)=\left\{\left[x_{1}, x_{2}\right] \mid\left(x_{1}, x_{2}\right) \in L^{2}\right.$ and $\left.x_{1} \leq_{L} x_{2}\right\}$. A (bilattice-based) triangle is a structure $\mathcal{I}(\mathcal{L})=\left(I(L), \leq_{t}, \leq_{k}\right)$, where, for every $\left[x_{1}, x_{2}\right],\left[y_{1}, y_{2}\right]$ in $I(L)$

1) $\left[x_{1}, x_{2}\right] \leq_{t}\left[y_{1}, y_{2}\right] \Leftrightarrow x_{1} \leq_{L} y_{1}$ and $x_{2} \leq_{L} y_{2}$;

2) $\left[x_{1}, x_{2}\right] \leq_{k}\left[y_{1}, y_{2}\right] \Leftrightarrow x_{1} \leq_{L} y_{1}$ and $x_{2} \geq_{L} y_{2}$.

Note that, in fact, a triangle $\mathcal{I}(\mathcal{L})$ is not a (pre-) bilattice, since the substructure $\left(I(L), \leq_{k}\right)$ is not a lattice (the supremum of 


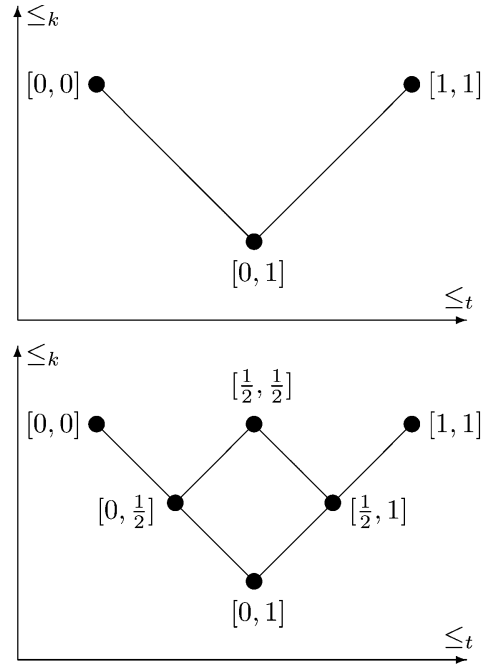

Fig. 2. Triangles $\mathcal{I}\left(\mathcal{L}_{2}\right)$ and $\mathcal{I}\left(\mathcal{L}_{3}\right)$.

any two elements does not necessarily exist). Still, triangles are very much in the same spirit as bilattices, since the $\leq_{k}$-ordering also represents differences in the amount of information that each interval exhibits. For this reason, $\mathcal{I}(\mathcal{L})$ is sometimes called a " $\leq_{k}$-lower prebilattice" [31] or a "weak interlaced bilattice" [32].

Example 2: The triangles $\mathcal{I}\left(\mathcal{L}_{2}\right)$ and $\mathcal{I}\left(\mathcal{L}_{3}\right)$ are shown in Fig. 2.

When $\mathcal{L}$ is the unit interval with the usual ordering, $\mathcal{I}(\mathcal{L})$ is a structure that corresponds to interval-valued fuzzy sets [19]. It follows, then, that triangles are generalized forms of these extensions of fuzzy sets (see again [17]).

We conclude this section by a result that relates the consistent elements of a given square and the associated triangle. ${ }^{2}$ First, we need some additional terminology and notations.

Definition 5: Let $\mathcal{B}=\left(B, \leq_{t}, \leq_{k}, \neg\right)$ be a bilattice with a conflation -. An element $x$ in $B$ is called exact with respect to this conflation if $x=-x$; it is consistent if $x \leq_{k}-x$.

Definition 6: Let - be a conflation of a bilattice $\mathcal{B}=\left(B, \leq_{t}\right.$, $\left.\leq_{k}, \neg\right)$. Denote by $\mathcal{C}^{-}(\mathcal{B})$ the substructure of $\mathcal{B}$ that consists of the consistent elements (with respect to - ) of $B$.

Proposition 2: [22], [17] Suppose that $\mathcal{L}$ is a complete lattice with an involution $\mathcal{N}$. Then $\mathcal{I}(\mathcal{L})$ is isomorphic to $\mathcal{C}^{-\mathcal{N}}\left(\mathcal{L}^{2}\right)$.

\section{Graded (BILATtice-Based) Logical Connectives}

In this section, which is the main part of this paper, we show how common extensions to $\mathcal{L}$-fuzzy set theory of the main connectives of classical logic can be related to bilattices in general, and to bilattice-based squares and triangles in particular. We divide this section to three parts, each one considers a basic connective (or two dual connectives, in case of Section III.B).

In what follows $\mathcal{L}=\left(L, \leq_{L}\right)$ is a complete lattice, and $0_{\mathcal{L}}=$ $\inf L, 1_{\mathcal{L}}=\sup L$.

\footnotetext{
${ }^{2}$ We refer to [16] and [17] for a further discussion on applications of this result in the context of fuzzy sets, and in particular its use for bridging between intuitionistic fuzzy set theory and interval fuzzy set theory.
}

\section{A. Negation}

Definition 7: A negator on $\mathcal{L}$ is any $\leq_{L}$-decreasing mapping $\mathcal{N}: L \rightarrow L$ such that $\mathcal{N}\left(0_{\mathcal{L}}\right)=1_{\mathcal{L}}$ and $\mathcal{N}\left(1_{\mathcal{L}}\right)=0_{\mathcal{L}}$. If for every $x, \mathcal{N}(\mathcal{N}(x))=x$, then $\mathcal{N}$ is an involutive negator on $\mathcal{L}$.

In the context of bilattices, the operation $\neg$ that appears in Definition 1 is an involutive negator on the lattice $\left(B, \leq_{t}\right)$. As a consequence, the operation $\neg$, defined on the square $\mathcal{L}^{2}$ by

$$
\neg\left(x_{1}, x_{2}\right)=\left(x_{2}, x_{1}\right),
$$

is an involutive negator on $\left(L^{2}, \leq_{t}\right)$. If a bilattice $\mathcal{B}$ has a conflation - , then by its definition this operation is an involutive negator on the lattice $\left(B, \leq_{k}\right)$.

Suppose now that $\mathcal{N}$ is an involutive negator on $\mathcal{L}$, i.e., every $x$ in $L$ has an $\leq_{L}$-involutive element $\mathcal{N}(x)$ in $L$. Then, as we noted before, a conflation $-\mathcal{N}$ of $\mathcal{L}^{2}$ may be defined by

$$
{ }_{\mathcal{N}}\left(x_{1}, x_{2}\right)=\left(\mathcal{N}\left(x_{2}\right), \mathcal{N}\left(x_{1}\right)\right) .
$$

In this case, another natural definition of a negator on $\left(L^{2}, \leq_{t}\right)$ is obtained by combining $\neg$ and $-_{\mathcal{N}}$, i.e., for every $\left(x_{1}, x_{2}\right)$ in $L^{23}$

$$
\sim_{\mathcal{N}}\left(x_{1}, x_{2}\right)=\neg-_{\mathcal{N}}\left(x_{1}, x_{2}\right)=\left(\mathcal{N}\left(x_{1}\right), \mathcal{N}\left(x_{2}\right)\right) .
$$

Note 1: One might wonder if there exist other 'interesting' negators apart from the prototypical ones described above. In [24], for the particular structure $\left([0,1]^{2}, \leq_{t}\right)$, it was shown that all involutive negators can be generated by simple transformations of the two basic choices $\neg$ and $\sim_{\mathcal{N}}$. The next proposition is a generalization of that result to squares.

Definition 8: For every element $x=\left(x_{1}, x_{2}\right)$ in $L^{2}$, define: $\operatorname{pr}_{1}(x)=x_{1}$ and $p r_{2}(x)=x_{2}$.

Proposition 3: Let $\mathcal{L}=\left(L, \leq_{L}\right)$ be a complete lattice that is a chain. An operation $\mathfrak{N}$ is an involutive negator on $\left(L^{2}, \leq_{t}\right)$ iff either

$$
\mathfrak{N}\left(x_{1}, x_{2}\right)=\left(\mathcal{N}_{1}\left(x_{1}\right), \mathcal{N}_{2}\left(x_{2}\right)\right)
$$

where $\mathcal{N}_{1}$ and $\mathcal{N}_{2}$ are two involutive negators on $\mathcal{L}$ such that $\mathcal{N}_{1}(x)=p r_{1} \mathfrak{N}\left(x, 0_{\mathcal{L}}\right)$ and $\mathcal{N}_{2}(x)=p r_{2} \mathfrak{N}\left(0_{\mathcal{L}}, x\right)$, or

$$
\mathfrak{N}\left(x_{1}, x_{2}\right)=\left(\varphi\left(x_{2}\right), \varphi^{-1}\left(x_{1}\right)\right)
$$

where $\varphi$ is an increasing permutation of $\mathcal{L}$ such that $\varphi(x)=$ $p r_{1} \mathfrak{N}\left(0_{\mathcal{L}}, x\right)$.

Proof: We shall show that if $\mathfrak{N}$ is an involutive negator on $\left(L^{2}, \leq_{t}\right)$ then either $\mathfrak{N}\left(0_{\mathcal{L}}, 0_{\mathcal{L}}\right)=\left(1_{\mathcal{L}}, 1_{\mathcal{L}}\right)$ or $\mathfrak{N}\left(0_{\mathcal{L}}, 0_{\mathcal{L}}\right)=$ $\left(0_{\mathcal{L}}, 0_{\mathcal{L}}\right)$ (Lemma 3-A); in the first case (1) applies (Lemmas 3-B,C,D) and in the second case (2) applies (Lemmas 3-E,F,G).

Lemma 3-A: Let $\mathcal{L}=\left(L, \leq_{L}\right)$ be a complete lattice that is a chain. For any involutive negator $\mathfrak{N}$ on $\left(L^{2}, \leq_{t}\right)$ it holds that either $\mathfrak{N}\left(0_{\mathcal{L}}, 0_{\mathcal{L}}\right)=\left(0_{\mathcal{L}}, 0_{\mathcal{L}}\right)$ or $\mathfrak{N}\left(0_{\mathcal{L}}, 0_{\mathcal{L}}\right)=\left(1_{\mathcal{L}}, 1_{\mathcal{L}}\right)$.

Proof: Assume first that $\mathfrak{N}\left(0_{\mathcal{L}}, 0_{\mathcal{L}}\right)=\left(x_{1}, 0_{\mathcal{L}}\right)$, where $x_{1} \quad>_{L} \quad 0_{\mathcal{L}}$. Then $\left(0_{\mathcal{L}}, 0_{\mathcal{L}}\right) \quad \leq_{t} \quad\left(x_{1}, 0_{\mathcal{L}}\right)$ and

${ }^{3}$ This operator has also been studied by Wagner [33], mainly in the context of four-valued logics, and by Doherty, Driankov, and Tsoukias [34] in the framework of DDT-logic (an operational version of Belnap's four-valued logic [27], [28]; see also [35]), where it is simply called "complement"). 
$\left(x_{1}, x_{1}\right) \quad \leq_{t} \quad\left(x_{1}, 0_{\mathcal{L}}\right)$, but $\left(0_{\mathcal{L}}, 0_{\mathcal{L}}\right)$ and $\left(x_{1}, x_{1}\right)$ are incomparable w.r.t. $\leq_{t}$. Since $\mathfrak{N}$ is decreasing and involutive, we obtain $\mathfrak{N}\left(0_{\mathcal{L}}, 0_{\mathcal{L}}\right) \geq_{t} \mathfrak{N}\left(x_{1}, 0_{\mathcal{L}}\right)=\left(0_{\mathcal{L}}, 0_{\mathcal{L}}\right)$ and $\mathfrak{N}\left(x_{1}, x_{1}\right) \geq_{t} \mathfrak{N}\left(x_{1}, 0_{\mathcal{L}}\right)=\left(0_{\mathcal{L}}, 0_{\mathcal{L}}\right)$. Hence, the second component of both $\mathfrak{N}\left(0_{\mathcal{L}}, 0_{\mathcal{L}}\right)$ and $\mathfrak{N}\left(x_{1}, x_{1}\right)$ must be $0_{\mathcal{L}}$, thus, $\mathfrak{N}\left(0_{\mathcal{L}}, 0_{\mathcal{L}}\right)$ and $\mathfrak{N}\left(x_{1}, x_{1}\right)$ are $\leq_{t}$-comparable. Now, since $\mathfrak{N}$ is decreasing and involutive, it also follows that $\left(0_{\mathcal{L}}, 0_{\mathcal{L}}\right)$ and $\left(x_{1}, x_{1}\right)$ are $\leq_{t}$-comparable, which is a contradiction.

Next, assume that $\mathfrak{N}\left(0_{\mathcal{L}}, 0_{\mathcal{L}}\right)=\left(1_{\mathcal{L}}, x_{2}\right)$, where $x_{2}<_{L}$ $1_{\mathcal{L}}$. Then $\left(1_{\mathcal{L}}, 1_{\mathcal{L}}\right) \leq_{t}\left(1_{\mathcal{L}}, x_{2}\right)$ and $\left(x_{2}, x_{2}\right) \leq_{t}\left(1_{\mathcal{L}}, x_{2}\right)$, but $\left(1_{\mathcal{L}}, 1_{\mathcal{L}}\right)$ and $\left(x_{2}, x_{2}\right)$ are $\leq_{t}$-incomparable. Similarly as above, a contradiction can be found.

When $\mathfrak{N}\left(0_{\mathcal{L}}, 0_{\mathcal{L}}\right)=\left(x_{1}, 1_{\mathcal{L}}\right)$, for $x_{1}<_{L} 1_{\mathcal{L}}$, we have that $\left(1_{\mathcal{L}}, 1_{\mathcal{L}}\right)$ and $\left(x_{1}, x_{1}\right)$ are two $\leq_{t}$-incomparable elements that are greater than or equal to $\left(x_{1}, 1_{\mathcal{L}}\right)$; likewise, if $\mathfrak{N}\left(0_{\mathcal{L}}, 0_{\mathcal{L}}\right)=$ $\left(0_{\mathcal{L}}, x_{2}\right)$, for $x_{2}>_{L} 0_{\mathcal{L}}$, we have that $\left(0_{\mathcal{L}}, 0_{\mathcal{L}}\right)$ and $\left(x_{2}, x_{2}\right)$ are two $\leq_{t}$-incomparable elements which are greater than or equal to $\left(0_{\mathcal{L}}, x_{2}\right)$. In both cases, a contradiction can be found in a similar way as previous cases.

Finally, assume that $\mathfrak{N}\left(0_{\mathcal{L}}, 0_{\mathcal{L}}\right)=\left(x_{1}, x_{2}\right)$, where $0_{\mathcal{L}}<_{L}$ $x_{1}<{ }_{L} 1_{\mathcal{L}}$ and $1_{\mathcal{L}}>_{L} x_{2}>_{L} 0_{\mathcal{L}}$. Then $\left(0_{\mathcal{L}}, x_{2}\right) \leq_{t}\left(x_{1}, x_{2}\right)$ and $\left(x_{1}, 1_{\mathcal{L}}\right) \leq_{t}\left(x_{1}, x_{2}\right)$, but $\left(0_{\mathcal{L}}, x_{2}\right)$ and $\left(x_{1}, 1_{\mathcal{L}}\right)$ are $\leq_{t}$-incomparable. Similarly as above, a contradiction can be found.

Summarizing, the only possible values of $\mathfrak{N}\left(0_{\mathcal{L}}, 0_{\mathcal{L}}\right)$ are $\left(0_{\mathcal{L}}, 0_{\mathcal{L}}\right)$ and $\left(1_{\mathcal{L}}, 1_{\mathcal{L}}\right)$.

Lemma 3-B: Let $\mathcal{L}=\left(L, \leq_{L}\right)$ be a complete lattice that is a chain. For any involutive negator $\mathfrak{N}$ on $\left(L^{2}, \leq_{t}\right)$ such that $\mathfrak{N}\left(0_{\mathcal{L}}, 0_{\mathcal{L}}\right)=\left(1_{\mathcal{L}}, 1_{\mathcal{L}}\right)$, the following holds for all $x_{1}, x_{2}$ in $L: \mathfrak{N}\left(x_{1}, 0_{\mathcal{L}}\right) \leq_{t}\left(1_{\mathcal{L}}, 1_{\mathcal{L}}\right), \mathfrak{N}\left(0_{\mathcal{L}}, x_{2}\right) \geq_{t}\left(1_{\mathcal{L}}, 1_{\mathcal{L}}\right)$, $\mathfrak{N}\left(x_{1}, 1_{\mathcal{L}}\right) \geq_{t}\left(0_{\mathcal{L}}, 0_{\mathcal{L}}\right)$ and $\mathfrak{N}\left(1_{\mathcal{L}}, x_{2}\right) \leq_{t}\left(0_{\mathcal{L}}, 0_{\mathcal{L}}\right)$.

Proof: Immediate from the fact that $\mathfrak{N}$ is an involutive negator and the assumption that $\mathfrak{N}\left(0_{\mathcal{L}}, 0_{\mathcal{L}}\right)=\left(1_{\mathcal{L}}, 1_{\mathcal{L}}\right)$.

Lemma 3-C: Let $\mathcal{L}=\left(L, \leq_{L}\right)$ be a complete lattice that is a chain. For any involutive negator $\mathfrak{N}$ on $\left(L^{2}, \leq_{t}\right)$ such that $\mathfrak{N}\left(0_{\mathcal{L}}, 0_{\mathcal{L}}\right)=\left(1_{\mathcal{L}}, 1_{\mathcal{L}}\right)$, it holds that $\operatorname{pr}_{1} \mathfrak{N}\left(x_{1}, x_{2}\right)=$ $p r_{1} \mathfrak{N}\left(x_{1}, 0_{\mathcal{L}}\right)$ and $p r_{2} \mathfrak{N}\left(x_{1}, x_{2}\right)=p r_{2} \mathfrak{N}\left(0_{\mathcal{L}}, x_{2}\right)$.

Proof: Let $x=\left(x_{1}, x_{2}\right)$ in $L^{2}$. We prove that $p r_{1} \mathfrak{N}\left(x_{1}, x_{2}\right)=p r_{1} \mathfrak{N}\left(x_{1}, 0_{\mathcal{L}}\right)$ (the proof that $p r_{2} \mathfrak{N}\left(x_{1}, x_{2}\right)=$ $p r_{2} \mathfrak{N}\left(0_{\mathcal{L}}, x_{2}\right)$ is completely analogous). When $x_{2}=0_{\mathcal{L}}$, the claim trivially holds; likewise, when $x_{1}=1_{\mathcal{L}}$, by Lemma 3-B and the fact that $\mathfrak{N}\left(1_{\mathcal{L}}, 0_{\mathcal{L}}\right)=\left(0_{\mathcal{L}}, 1_{\mathcal{L}}\right)$ it holds that $p r_{1} \mathfrak{N}\left(x_{1}, x_{2}\right)=0_{\mathcal{L}}=p r_{1} \mathfrak{N}\left(x_{1}, 0_{\mathcal{L}}\right)$.

It remains to consider the case in which $x_{2}>_{L} 0_{\mathcal{L}}$ and $x_{1}<_{L} 1_{\mathcal{L}}$. Since $\mathfrak{N}$ is decreasing, $\mathfrak{N}\left(x_{1}, x_{2}\right) \geq_{t} \mathfrak{N}\left(x_{1}, 0_{\mathcal{L}}\right)$, so if we suppose for a contradiction that $\operatorname{pr}_{1} \mathfrak{N}\left(x_{1}, x_{2}\right) \neq$ $p r_{1} \mathfrak{N}\left(x_{1}, 0_{\mathcal{L}}\right)$, we have that $p r_{1} \mathfrak{N}\left(x_{1}, x_{2}\right)>_{L} p r_{1} \mathfrak{N}\left(x_{1}, 0_{\mathcal{L}}\right)$. Note also that by Lemma 3-B, $p r_{2} \mathfrak{N}\left(x_{1}, 0_{\mathcal{L}}\right)=1_{\mathcal{L}}$. We distinguish between the following two cases.

- If $p r_{2} \mathfrak{N}\left(x_{1}, x_{2}\right)=1_{\mathcal{L}}$, then by Lemma 3-B, it follows that $x_{2}=p r_{2} \mathfrak{N}\left(\mathfrak{N}\left(x_{1}, x_{2}\right)\right)=0_{\mathcal{L}}$, which is a contradiction.

- If $\operatorname{pr}_{2} \mathfrak{N}\left(x_{1}, x_{2}\right)<_{L} 1_{\mathcal{L}}$, then let $b=\left(b_{1}, b_{2}\right)=$ $\left(p r_{1} \mathfrak{N}\left(x_{1}, x_{2}\right), 1_{\mathcal{L}}\right)$ and $c=\left(c_{1}, c_{2}\right)=\left(p r_{1} \mathfrak{N}\left(x_{1}, 0_{\mathcal{L}}\right)\right.$, $\left.p r_{2} \mathfrak{N}\left(x_{1}, x_{2}\right)\right)$. It is easy to check, then, that $b$ and $c$ are $\leq_{t}$-incomparable. Moreover, it can be verified that $\mathfrak{N}\left(x_{1}, 0_{\mathcal{L}}\right) \quad \leq_{t} \quad b \quad \leq_{t} \quad \mathfrak{N}\left(x_{1}, x_{2}\right)$ and $\mathfrak{N}\left(x_{1}, 0_{\mathcal{L}}\right) \leq_{t} c \leq_{t} \mathfrak{N}\left(x_{1}, x_{2}\right)$. Since $\mathfrak{N}$ is decreasing, we obtain that $\left(x_{1}, x_{2}\right) \leq_{t} \mathfrak{N}(b) \leq_{t}\left(x_{1}, 0_{\mathcal{L}}\right)$ and $\left(x_{1}, x_{2}\right) \leq_{t}$ $\mathfrak{N}(c) \leq_{t}\left(x_{1}, 0_{\mathcal{L}}\right)$. Thus, $p r_{1} \mathfrak{N}(b)=p r_{1} \mathfrak{N}(c)=x_{1}$, so $\mathfrak{N}(b)$ and $\mathfrak{N}(c)$ are $\leq_{t}$-comparable. But since $\mathfrak{N}$ is decreasing and involutive, this would mean that $b$ and $c$ are also $\leq_{t}$-comparable, which is a contradiction.

Lemma 3-D: Let $\mathcal{L}=\left(L, \leq_{L}\right)$ be a complete lattice that is a chain and let $\mathfrak{N}$ be a negator on $\left(\mathcal{L}^{2}, \leq_{t}\right)$ such that $\mathfrak{N}\left(0_{\mathcal{L}}, 0_{\mathcal{L}}\right)=$ $\left(1_{\mathcal{L}}, 1_{\mathcal{L}}\right)$. Furthermore, let $\mathcal{N}_{1}$ and $\mathcal{N}_{2}$ be the $L \rightarrow L$ mappings defined by $\mathcal{N}_{1}(x)=p r_{1} \mathfrak{N}\left(x, 0_{\mathcal{L}}\right)$ and $\mathcal{N}_{2}(x)=p r_{2} \mathfrak{N}\left(0_{\mathcal{L}}, x\right)$. Then $\mathfrak{N}$ is involutive if and only if $\mathcal{N}_{1}$ and $\mathcal{N}_{2}$ are involutive negators on $\mathcal{L}$, and for all $\left(x_{1}, x_{2}\right)$ in $L^{2}$

$$
\mathfrak{N}\left(x_{1}, x_{2}\right)=\left(\mathcal{N}_{1}\left(x_{1}\right), \mathcal{N}_{2}\left(x_{2}\right)\right) .
$$

Proof: Assume first that $\mathfrak{N}$ is an involutive negator on $\left(\mathcal{L}^{2}, \leq_{t}\right)$ such that $\mathfrak{N}\left(0_{\mathcal{L}}, 0_{\mathcal{L}}\right)=\left(1_{\mathcal{L}}, 1_{\mathcal{L}}\right)$. Define the $L \rightarrow L$ mappings $\mathcal{N}_{1}$ and $\mathcal{N}_{2}$ by

$$
\begin{aligned}
& \mathcal{N}_{1}(x)=p r_{1} \mathfrak{N}\left(x, 0_{\mathcal{L}}\right) \\
& \mathcal{N}_{2}(x)=p r_{2} \mathfrak{N}\left(0_{\mathcal{L}}, x\right) .
\end{aligned}
$$

It is clear that $\mathcal{N}_{1}\left(0_{\mathcal{L}}\right)=\mathcal{N}_{2}\left(0_{\mathcal{L}}\right)=1_{\mathcal{L}}$ and $\mathcal{N}_{1}\left(1_{\mathcal{L}}\right)=$ $\mathcal{N}_{2}\left(1_{\mathcal{L}}\right)=0_{\mathcal{L}}$. Moreover, since $\mathfrak{N}$ is decreasing, so are $\mathcal{N}_{1}$ and $\mathcal{N}_{2}$. Hence, $\mathcal{N}_{1}$ and $\mathcal{N}_{2}$ are negators on $\mathcal{L}$.

By Lemma 3-C, $p r_{1} \mathfrak{N}\left(x_{1}, x_{2}\right)=p r_{1} \mathfrak{N}\left(x_{1}, 0_{\mathcal{L}}\right)=\mathcal{N}_{1}\left(x_{1}\right)$ and $p r_{2} \mathfrak{N}\left(x_{1}, x_{2}\right)=p r_{2} \mathfrak{N}\left(0_{\mathcal{L}}, x_{2}\right)=\mathcal{N}_{2}\left(x_{2}\right)$. In other words, $\mathfrak{N}\left(x_{1}, x_{2}\right)=\left(\mathcal{N}_{1}\left(x_{1}\right), \mathcal{N}_{2}\left(x_{2}\right)\right)$. Moreover, since $\mathfrak{N}$ is involutive, we obtain $\left(x_{1}, x_{2}\right)=\mathfrak{N}\left(\mathfrak{N}\left(x_{1}, x_{2}\right)\right)=$ $\left(\mathcal{N}_{1}\left(\mathcal{N}_{1}\left(x_{1}\right)\right), \mathcal{N}_{2}\left(\mathcal{N}_{2}\left(x_{2}\right)\right)\right)$, so $\mathcal{N}_{1}$ and $\mathcal{N}_{2}$ are involutive.

Assume conversely that $\mathcal{N}_{1}$ and $\mathcal{N}_{2}$ are involutive negators on $\mathcal{L}$ and define the mapping $\mathfrak{N}: L^{2} \rightarrow L^{2}$ by

$$
\mathfrak{N}\left(x_{1}, x_{2}\right)=\left(\mathcal{N}_{1}\left(x_{1}\right), \mathcal{N}_{2}\left(x_{2}\right)\right) .
$$

Then clearly $\mathfrak{N}\left(0_{\mathcal{L}}, 0_{\mathcal{L}}\right)=\left(1_{\mathcal{L}}, 1_{\mathcal{L}}\right), \mathfrak{N}\left(1_{\mathcal{L}}, 0_{\mathcal{L}}\right)=\left(0_{\mathcal{L}}, 1_{\mathcal{L}}\right)$ and $\mathfrak{N}\left(0_{\mathcal{L}}, 1_{\mathcal{L}}\right)=\left(1_{\mathcal{L}}, 0_{\mathcal{L}}\right)$. Also, since $\mathcal{N}_{1}$ and $\mathcal{N}_{2}$ are decreasing, so is $\mathfrak{N}$. Moreover, $\mathfrak{N}\left(\mathfrak{N}\left(x_{1}, x_{2}\right)\right)=\left(\mathcal{N}_{1}\left(\mathcal{N}_{1}\left(x_{1}\right)\right)\right.$, $\left.\mathcal{N}_{2}\left(\mathcal{N}_{2}\left(x_{2}\right)\right)\right)=\left(x_{1}, x_{2}\right)$, so $\mathfrak{N}$ is an involutive negator on $\left(\mathcal{L}^{2}, \leq_{t}\right)$.

Lemma 3-E: Let $\mathcal{L}=\left(L, \leq_{L}\right)$ be a complete lattice that is a chain. For any involutive negator $\mathfrak{N}$ on $\left(L^{2}, \leq_{t}\right)$ such that $\mathfrak{N}\left(0_{\mathcal{L}}, 0_{\mathcal{L}}\right)=\left(0_{\mathcal{L}}, 0_{\mathcal{L}}\right)$, the following holds for all $x_{1}, x_{2}$ in $L: \mathfrak{N}\left(x_{1}, 0_{\mathcal{L}}\right) \quad \leq_{t}\left(0_{\mathcal{L}}, 0_{\mathcal{L}}\right), \mathfrak{N}\left(0_{\mathcal{L}}, x_{2}\right) \geq_{t}\left(0_{\mathcal{L}}, 0_{\mathcal{L}}\right)$, $\mathfrak{N}\left(x_{1}, 1_{\mathcal{L}}\right) \geq_{t}\left(1_{\mathcal{L}}, 1_{\mathcal{L}}\right)$ and $\mathfrak{N}\left(1_{\mathcal{L}}, x_{2}\right) \leq_{t}\left(1_{\mathcal{L}}, 1_{\mathcal{L}}\right)$.

Proof: Immediate from the involutivity of $\mathfrak{N}$, the assumption that $\mathfrak{N}\left(0_{\mathcal{L}}, 0_{\mathcal{L}}\right)=\left(0_{\mathcal{L}}, 0_{\mathcal{L}}\right)$ and the fact that under that assumption also $\mathfrak{N}\left(1_{\mathcal{L}}, 1_{\mathcal{L}}\right)=\left(1_{\mathcal{L}}, 1_{\mathcal{L}}\right)$.

Lemma 3-F: Let $\mathcal{L}=\left(L, \leq_{L}\right)$ be a complete lattice that is a chain. For any involutive negator $\mathfrak{N}$ on $\left(L^{2}, \leq_{t}\right)$ such that $\mathfrak{N}\left(0_{\mathcal{L}}, 0_{\mathcal{L}}\right)=\left(0_{\mathcal{L}}, 0_{\mathcal{L}}\right)$, it holds that $\operatorname{pr}_{1} \mathfrak{N}\left(x_{1}, x_{2}\right)=$ $p r_{1} \mathfrak{N}\left(0_{\mathcal{L}}, x_{2}\right)$ and $p r_{2} \mathfrak{N}\left(x_{1}, x_{2}\right)=p r_{2} \mathfrak{N}\left(x_{1}, 0_{\mathcal{L}}\right)$ for all $\left(x_{1}, x_{2}\right)$ in $L^{2}$.

Proof: Let $x=\left(x_{1}, x_{2}\right)$ be an element in $L^{2}$. We prove that $\operatorname{pr}_{1} \mathfrak{N}\left(x_{1}, x_{2}\right)=p r_{1} \mathfrak{N}\left(0_{\mathcal{L}}, x_{2}\right)$ (the proof that $p r_{2} \mathfrak{N}\left(x_{1}, x_{2}\right)=p r_{2} \mathfrak{N}\left(x_{1}, 0_{\mathcal{L}}\right)$ is completely analogous). When $x_{1}=0_{\mathcal{L}}$, the claim trivially holds; likewise it is true when $x_{2}=1_{\mathcal{L}}$, by Lemma 3 -E and the fact that $\mathfrak{N}\left(0_{\mathcal{L}}, 1_{\mathcal{L}}\right)=\left(1_{\mathcal{L}}, 0_{\mathcal{L}}\right)$. Assume now that $x_{1}>_{L} 0_{\mathcal{L}}$, $x_{2}<_{L} 1_{\mathcal{L}}$ and $p r_{1} \mathfrak{N}\left(x_{1}, x_{2}\right) \neq p r_{1} \mathfrak{N}\left(0_{\mathcal{L}}, x_{2}\right)$. Then, since $\mathfrak{N}$ is decreasing, $p r_{1} \mathfrak{N}\left(x_{1}, x_{2}\right)<_{L} p r_{1} \mathfrak{N}\left(0_{\mathcal{L}}, x_{2}\right)$. Note also that 
by Lemma 3-E, $p r_{2} \mathfrak{N}\left(0_{\mathcal{L}}, x_{2}\right)=0_{\mathcal{L}}$. We distinguish between the following two cases.

- If $p r_{2} \mathfrak{N}\left(x_{1}, x_{2}\right)=0_{\mathcal{L}}$, then by Lemma 3-E, it follows that $x_{1}=\operatorname{pr}_{1} \mathfrak{N}\left(\mathfrak{N}\left(x_{1}, x_{2}\right)\right)=0_{\mathcal{L}}$, which is a contradiction.

- If $\operatorname{pr}_{2} \mathfrak{N}\left(x_{1}, x_{2}\right) \quad>_{L} 0_{\mathcal{L}}$, then let $b=\left(b_{1}, b_{2}\right)=$ $\left(p r_{1} \mathfrak{N}\left(0_{\mathcal{L}}, x_{2}\right), p r_{2} \mathfrak{N}\left(x_{1}, x_{2}\right)\right)$ and $c=\left(c_{1}, c_{2}\right)=$ $\left(p r_{1} \mathfrak{N}\left(x_{1}, x_{2}\right), 0_{\mathcal{L}}\right)$. It is easy to check, then, that $b$ and $c$ are $\leq_{t}$-incomparable. Moreover, it can be verified that $\mathfrak{N}\left(0_{\mathcal{L}}, x_{2}\right) \quad \geq_{t} \quad b \quad \geq_{t} \quad \mathfrak{N}\left(x_{1}, x_{2}\right)$ and $\mathfrak{N}\left(0_{\mathcal{L}}, x_{2}\right) \geq_{t} c \geq_{t} \mathfrak{N}\left(x_{1}, x_{2}\right)$. Since $\mathfrak{N}$ is decreasing, we obtain that $\left(x_{1}, x_{2}\right) \geq_{t} \mathfrak{N}(b) \geq_{t}\left(0_{\mathcal{L}}, x_{2}\right)$ and $\left(x_{1}, x_{2}\right) \geq_{t}$ $\mathfrak{N}(c) \geq_{t}\left(0_{\mathcal{L}}, x_{2}\right)$. Thus, $p r_{2} \mathfrak{N}(b)=p r_{2} \mathfrak{N}(c)=x_{2}$, so $\mathfrak{N}(b)$ and $\mathfrak{N}(c)$ are $\leq_{t}$-comparable. But since $\mathfrak{N}$ is decreasing and involutive, this would mean that $b$ and $c$ are also $\leq_{t}$-comparable, which is a contradiction.

Lemma 3-G: Let $\mathcal{L}=\left(L, \leq_{L}\right)$ be a complete lattice that is a chain and let $\mathfrak{N}$ be a negator on $\left(L^{2}, \leq_{t}\right)$ such that $\mathfrak{N}\left(0_{\mathcal{L}}, 0_{\mathcal{L}}\right)=$ $\left(0_{\mathcal{L}}, 0_{\mathcal{L}}\right)$. Furthermore, let $\varphi$ be the $L \rightarrow L$ mapping defined by $\varphi(x)=p r_{1} \mathfrak{N}\left(0_{\mathcal{L}}, x\right)$. Then $\mathfrak{N}$ is involutive if and only if $\varphi$ is an $\leq_{L}$-increasing permutation of $L$, and for all $\left(x_{1}, x_{2}\right)$ in $L^{2}$,

$$
\mathfrak{N}\left(x_{1}, x_{2}\right)=\left(\varphi\left(x_{2}\right), \varphi^{-1}\left(x_{1}\right)\right) .
$$

Proof: Assume first that $\mathfrak{N}$ is an involutive negator on $\left(\mathcal{L}^{2}, \leq_{t}\right)$ such that $\mathfrak{N}\left(0_{\mathcal{L}}, 0_{\mathcal{L}}\right)=\left(0_{\mathcal{L}}, 0_{\mathcal{L}}\right)$. Define the $L \rightarrow L$ mappings $\varphi_{1}$ and $\varphi_{2}$ by

$$
\begin{aligned}
\varphi_{1}(x) & =p r_{1} \mathfrak{N}\left(0_{\mathcal{L}}, x\right) \\
\varphi_{2}(x) & =p r_{2} \mathfrak{N}\left(x, 0_{\mathcal{L}}\right)
\end{aligned}
$$

It is clear that $\varphi_{1}\left(0_{\mathcal{L}}\right)=\varphi_{2}\left(0_{\mathcal{L}}\right)=0_{\mathcal{L}}$ and $\varphi_{1}\left(1_{\mathcal{L}}\right)=$ $\varphi_{2}\left(1_{\mathcal{L}}\right)=1_{\mathcal{L}}$. Also, since $\mathfrak{N}$ is decreasing, $\varphi_{1}$ and $\varphi_{2}$ are increasing. Moreover, due to the fact that $\mathfrak{N}$ is an involutive negator, and since Lemma 3-E asserts that $p r_{2} \mathfrak{N}\left(0_{\mathcal{L}}, x\right)=p r_{1} \mathfrak{N}\left(x, 0_{\mathcal{L}}\right)=0_{\mathcal{L}}$, it holds that $\varphi_{1}$ and $\varphi_{2}$ are permutations of $L$ (i.e., bijections from $L$ to itself).

By Lemma 3-F, for every element $\left(x_{1}, x_{2}\right)$ in $L^{2}$ it holds that $\operatorname{pr}_{1} \mathfrak{N}\left(x_{1}, x_{2}\right)=p r_{1} \mathfrak{N}\left(0_{\mathcal{L}}, x_{2}\right)=\varphi_{1}\left(x_{2}\right)$ and $p r_{2} \mathfrak{N}\left(x_{1}, x_{2}\right)=p r_{2} \mathfrak{N}\left(x_{1}, 0_{\mathcal{L}}\right)=\varphi_{2}\left(x_{1}\right)$. In other words, for every $\left(x_{1}, x_{2}\right)$ in $L^{2}, \mathfrak{N}\left(x_{1}, x_{2}\right)=\left(\varphi_{1}\left(x_{2}\right), \varphi_{2}\left(x_{1}\right)\right)$. Moreover, since $\mathfrak{N}$ is involutive, we have that $\mathfrak{N}\left(\mathfrak{N}\left(x_{1}, x_{2}\right)\right)=$ $\left(\varphi_{1}\left(\varphi_{2}\left(x_{1}\right)\right), \varphi_{2}\left(\varphi_{1}\left(x_{2}\right)\right)\right)=\left(x_{1}, x_{2}\right)$, hence, $\varphi_{2}=\varphi_{1}^{-1}$.

Assume conversely that $\varphi$ is an increasing permutation of $L$ and define the mapping $\mathfrak{N}: L^{2} \rightarrow L^{2}$ as follows:

$$
\mathfrak{N}\left(x_{1}, x_{2}\right)=\left(\varphi\left(x_{2}\right), \varphi^{-1}\left(x_{1}\right)\right) .
$$

Clearly, $\mathfrak{N}\left(0_{\mathcal{L}}, 0_{\mathcal{L}}\right)=\left(0_{\mathcal{L}}, 0_{\mathcal{L}}\right), \mathfrak{N}\left(0_{\mathcal{L}}, 1_{\mathcal{L}}\right)=\left(1_{\mathcal{L}}, 0_{\mathcal{L}}\right)$ and $\mathfrak{N}\left(1_{\mathcal{L}}, 0_{\mathcal{L}}\right)=\left(0_{\mathcal{L}}, 1_{\mathcal{L}}\right)$. Since $\varphi$ is increasing, $\mathfrak{N}$ is decreasing. Moreover, $\mathfrak{N}\left(\mathfrak{N}\left(x_{1}, x_{2}\right)\right)=\left(\varphi\left(\varphi^{-1}\left(x_{1}\right)\right), \varphi^{-1}\left(\varphi\left(x_{2}\right)\right)\right)=$ $\left(x_{1}, x_{2}\right)$, so $\mathfrak{N}$ is an involutive negator on $\left(\mathcal{L}^{2}, \leq_{t}\right)$.

This completes the Proof of Lemma 3-G and Proposition 3.

Clearly, $\sim_{\mathcal{N}}$ is obtained from Proposition 3 by (1) where $\mathcal{N}=\mathcal{N}_{1}=\mathcal{N}_{2}$, and $\neg$ is obtained from (2) where $\varphi$ is the identity permutation of $L$. One of the advantages of $\sim_{\mathcal{N}}$ is that it preserves the following weakened version of the law of the excluded middle.
Definition 9: An involutive negator $\mathcal{N}$ on $\mathcal{L}$ is called Kleene negator, if for all $x, y$ in $L$

$$
x \wedge_{L} \mathcal{N}(x) \leq_{L} y \vee_{L} \mathcal{N}(y) .
$$

The intuition behind Kleene's condition is that even in a context where excluded middle and/or the contradiction law do not hold, "intended" contradictions should still not surpass "intended" tautologies with respect to the truth order. The following claim is easily verified:

Proposition 4: If $\mathcal{N}$ is a Kleene negator on $\mathcal{L}$, then $\sim_{\mathcal{N}}$ is a Kleene negator on $\left(L^{2}, \leq_{t}\right)$.

Unlike $\sim_{\mathcal{N}}$, the negator $\neg$ never satisfies Kleene's condition. To see this, consider, for instance, $\left(x_{1}, x_{2}\right)=\left(1_{\mathcal{L}}, 1_{\mathcal{L}}\right)$ and $\left(y_{1}, y_{2}\right)=\left(0_{\mathcal{L}}, 0_{\mathcal{L}}\right)$. Then

$$
\begin{aligned}
\left(x_{1}, x_{2}\right) \wedge \neg\left(x_{1}, x_{2}\right) & =\left(1_{\mathcal{L}}, 1_{\mathcal{L}}\right) \\
& \swarrow_{t}\left(0_{\mathcal{L}}, 0_{\mathcal{L}}\right) \\
& =\left(y_{1}, y_{2}\right) \vee \neg\left(y_{1}, y_{2}\right) .
\end{aligned}
$$

On the other hand, $\sim_{\mathcal{N}}$ also has some disadvantages. For instance, it cannot serve as a (bilattice) negation on $\mathcal{L}^{2}$ in the sense of Definition 1, since it does not preserve the $\leq_{k}$-order. Indeed, in $\mathcal{L}_{2}^{2}$, for example, although $(1,0) \leq_{k}(1,1)$, still $\sim_{\mathcal{N}}(1,0)=(0,1) \quad \mathbb{Z}_{k}(0,0)=\sim_{\mathcal{N}}(1,1)$.

Consider now negators in triangles $\mathcal{I}(\mathcal{L})$, or-equivalently (see Proposition 2) -in the substructure $\mathcal{C}^{-\mathcal{N}}(\mathcal{L})$ of the consistent elements in the square $\mathcal{L}^{2}$. As the following proposition shows, it is rather straightforward to find an analogous definition of $\neg$ for these structures, while for $\sim_{\mathcal{N}}$ this is not possible.

Proposition 5: Let $\mathcal{L}$ be a complete lattice with an involutive negator $\mathcal{N}$. Then $\mathcal{C}^{-\mathcal{N}}(\mathcal{L})$ is closed under $\neg$ but not under $\sim_{\mathcal{N}}$.

Proof: For the first part of the claim we have to show that if $\left(x_{1}, x_{2}\right)$ in $\mathcal{C}^{-\mathcal{N}}(\mathcal{L})$, so is $\neg\left(x_{1}, x_{2}\right)$. Indeed, $\left(x_{1}, x_{2}\right) \quad \leq_{k}-{ }_{\mathcal{N}}\left(x_{1}, x_{2}\right)=\left(\mathcal{N}\left(x_{2}\right), \mathcal{N}\left(x_{1}\right)\right)$ and so $x_{1} \leq_{L} \quad \mathcal{N}\left(x_{2}\right)$ and $x_{2} \leq_{L} \quad \mathcal{N}\left(x_{1}\right)$. Thus, $\neg\left(x_{1}, x_{2}\right)=$ $\left(x_{2}, x_{1}\right) \leq_{k}\left(\mathcal{N}\left(x_{1}\right), \mathcal{N}\left(x_{2}\right)\right)=-\mathcal{N}\left(x_{2}, x_{1}\right)=-_{\mathcal{N}} \neg\left(x_{1}, x_{2}\right)$.

For the second part of the claim, consider, e.g., $\left(0_{\mathcal{L}}, 0_{\mathcal{L}}\right)$. Then $\left(0_{\mathcal{L}}, 0_{\mathcal{L}}\right)$ is in $\mathcal{C}^{-\mathcal{N}}(\mathcal{L})$, since $\left(0_{\mathcal{L}}, 0_{\mathcal{L}}\right)<_{k}\left(1_{\mathcal{L}}, 1_{\mathcal{L}}\right)=$ $-_{\mathcal{N}}\left(0_{\mathcal{L}}, 0_{\mathcal{L}}\right)$. On the other hand, $\sim_{\mathcal{N}}\left(0_{\mathcal{L}}, 0_{\mathcal{L}}\right)=\left(1_{\mathcal{L}}, 1_{\mathcal{L}}\right) \notin$ $\mathcal{C}^{-\mathcal{N}}(\mathcal{L})$, since $\left(1_{\mathcal{L}}, 1_{\mathcal{L}}\right) \Varangle_{k}\left(0_{\mathcal{L}}, 0_{\mathcal{L}}\right)=-_{\mathcal{N}}\left(1_{\mathcal{L}}, 1_{\mathcal{L}}\right)$.

Thus, for the negator $\neg$, a corresponding triangle operation may be obtained by applying the isomorphism $f\left(\left[x_{1}, x_{2}\right]\right)=$ $\left(x_{1}, \mathcal{N}\left(x_{2}\right)\right)$, used in [22] for the proof of Proposition 2, to obtain an operation $\mathfrak{N}$ defined, for every $\left[x_{1}, x_{2}\right]$ in $I(L)$, by

$$
\mathfrak{N}\left(\left[x_{1}, x_{2}\right]\right)=\left[\mathcal{N}\left(x_{2}\right), \mathcal{N}\left(x_{1}\right)\right] .
$$

Clearly, $\left[\mathcal{N}\left(x_{2}\right), \mathcal{N}\left(x_{1}\right)\right]$ is an interval, and so $\mathfrak{N}$ is an involutive negator on $\left(I(L), \leq_{t}\right)$.

Next we show, as we did for squares (cf. Proposition 3), that (3) is a characterization of involutive negators in many common triangles.

Definition 10: For every interval $x=\left[x_{1}, x_{2}\right]$ in $I(L)$, denote: $l(x)=x_{1}$ and $r(x)=x_{2}$. 
Definition 11: Denote by $] a, b]$ (respectively, $[a, b[$ ) the interval $[a, b]$ without $a$ (respectively, without $b$ ). Denote by $] a, b[$ the interval without $a$ and $b$.

Proposition 6: Let $\mathcal{L}=\left(L, \leq_{L}\right)$ be a complete lattice that is a chain with at least four elements. An operation $\mathfrak{N}$ is an involutive negator on $\left(I(L), \leq_{t}\right)$ iff

$$
\mathfrak{N}\left(\left[x_{1}, x_{2}\right]\right)=\left[\mathcal{N}\left(x_{2}\right), \mathcal{N}\left(x_{1}\right)\right]
$$

where $\mathcal{N}$ is an involutive negator on $\mathcal{L}$, such that $\mathcal{N}(x)=$ $r\left(\mathfrak{N}\left[x, 1_{\mathcal{L}}\right]\right)=l\left(\mathfrak{N}\left[0_{\mathcal{L}}, x\right]\right)$.

Proof: In the proof we shall use the following notation: $D=\{[x, x] \mid x \in L\}$.

Lemma 6-A: Let $\mathcal{L}=\left(L, \leq_{L}\right)$ be a complete lattice that is a chain with at least four elements. For any involutive negator $\mathfrak{N}$ on $\left(I(L), \leq_{t}\right)$ it holds that $\mathfrak{N}\left(\left[0_{\mathcal{L}}, 1_{\mathcal{L}}\right]\right)=\left[0_{\mathcal{L}}, 1_{\mathcal{L}}\right]$.

Proof: Assume first that $\mathfrak{N}\left(\left[0_{\mathcal{L}}, 1_{\mathcal{L}}\right]\right)=\left[x_{1}, 1_{\mathcal{L}}\right]$, where $\left.\left.x_{1} \quad \in\right] 0_{\mathcal{L}}, 1_{\mathcal{L}}\right]$. Then $\left[0_{\mathcal{L}}, 1_{\mathcal{L}}\right] \quad \leq_{t} \quad\left[x_{1}, 1_{\mathcal{L}}\right]$ and $\left[x_{1}^{\prime}, x_{1}^{\prime}\right] \leq_{t}\left[x_{1}, 1_{\mathcal{L}}\right]$, where $0_{\mathcal{L}}<_{L} x_{1}^{\prime}<_{L} 1_{\mathcal{L}}$ if $x_{1}=1_{\mathcal{L}}$ (such an $x_{1}^{\prime}$ exists since there are at least four elements in $L$ ) and $x_{1}^{\prime}=x_{1}$ otherwise. Note that $\left[0_{\mathcal{L}}, 1_{\mathcal{L}}\right]$ and $\left[x_{1}^{\prime}, x_{1}^{\prime}\right]$ are incomparable w.r.t. $\leq_{t}$. Since $\mathfrak{N}$ is decreasing and involutive, $\mathfrak{N}\left(\left[0_{\mathcal{L}}, 1_{\mathcal{L}}\right]\right) \geq_{t} \mathfrak{N}\left(\left[x_{1}, 1_{\mathcal{L}}\right]\right)=\left[0_{\mathcal{L}}, 1_{\mathcal{L}}\right]$ and $\mathfrak{N}\left(\left[x_{1}^{\prime}, x_{1}^{\prime}\right]\right) \geq_{t}$ $\left[0_{\mathcal{L}}, 1_{\mathcal{L}}\right]$, so $r\left(\mathfrak{N}\left(\left[0_{\mathcal{L}}, 1_{\mathcal{L}}\right]\right)\right)=r\left(\mathfrak{N}\left(\left[x_{1}^{\prime}, x_{1}^{\prime}\right]\right)\right)=1_{\mathcal{L}}$. Hence, $\mathfrak{N}\left(\left[0_{\mathcal{L}}, 1_{\mathcal{L}}\right]\right)$ and $\mathfrak{N}\left(\left[x_{1}^{\prime}, x_{1}^{\prime}\right]\right)$ are $\leq_{t}$-comparable. Since $\mathfrak{N}$ is decreasing and involutive, it follows that $\left[0_{\mathcal{L}}, 1_{\mathcal{L}}\right]$ and $\left[x_{1}^{\prime}, x_{1}^{\prime}\right]$ are $\leq_{t}$-comparable, which is a contradiction.

Assume now that $\mathfrak{N}\left(\left[0_{\mathcal{L}}, 1_{\mathcal{L}}\right]\right)=\left[x_{1}, x_{2}\right]$, where $x_{2}<1_{\mathcal{L}}$. If $x_{1}=x_{2}$ and $\sup \left(L \backslash\left\{1_{\mathcal{L}}\right\}\right)=x_{2}$, then, since there are at least four elements in $L$, there exists an $\left.x_{2}^{\prime} \in\right] 0_{\mathcal{L}}, x_{2}\left[\right.$. Thus, $\left[0_{\mathcal{L}}, x_{2}\right]$ and $\left[x_{2}^{\prime}, x_{2}^{\prime}\right]$ are incomparable with respect to $\leq_{t}$, but both are smaller than or equal to $\left[x_{2}, x_{2}\right]$. Since $\mathfrak{N}$ is decreasing, we obtain $\mathfrak{N}\left(\left[0_{\mathcal{L}}, x_{2}\right]\right) \geq_{t}\left[0_{\mathcal{L}}, 1_{\mathcal{L}}\right]$ and $\mathfrak{N}\left(\left[x_{2}^{\prime}, x_{2}^{\prime}\right]\right) \geq_{t}\left[0_{\mathcal{L}}, 1_{\mathcal{L}}\right]$. Similarly as above, we find a contradiction.

If $x_{1}<_{L} x_{2}$, then $\left[x_{1}, 1_{\mathcal{L}}\right]$ and $\left[x_{2}, x_{2}\right]$ are incomparable with respect to $\leq_{t}$, but both are greater than $\left[x_{1}, x_{2}\right]$. Since $\mathfrak{N}$ is decreasing and involutive, it follows $\mathfrak{N}\left(\left[x_{1}, 1_{\mathcal{L}}\right]\right) \leq_{t}\left[0_{\mathcal{L}}, 1_{\mathcal{L}}\right]$ and $\mathfrak{N}\left(\left[x_{2}, x_{2}\right]\right) \leq_{t}\left[0_{\mathcal{L}}, 1_{\mathcal{L}}\right]$. Similarly as above, we find a contradiction.

If $x_{1}=x_{2}$, but $\sup \left(L \backslash\left\{1_{\mathcal{L}}\right\}\right)>_{L} x_{2}$, then similarly as in the previous case, two incomparable elements can be found which are greater than $\left[x_{1}, x_{2}\right]$, and similarly a contradiction can be obtained.

Corollary 6-B: Let $\mathcal{L}=\left(L, \leq_{L}\right)$ be a complete lattice that is a chain with at least four elements. For any involutive negator $\mathfrak{N}$ on $\left(I(L), \leq_{t}\right)$ it holds that $\mathfrak{N}\left(\left[0_{\mathcal{L}}, x_{2}\right]\right) \geq_{t}\left[0_{\mathcal{L}}, 1_{\mathcal{L}}\right]$ and $\mathfrak{N}\left(\left[x_{1}, 1_{\mathcal{L}}\right]\right) \leq_{t}\left[0_{\mathcal{L}}, 1_{\mathcal{L}}\right]$, for all $x_{1}, x_{2}$ in $\left[0_{\mathcal{L}}, 1_{\mathcal{L}}\right]$.

Lemma 6-C: Let $\mathcal{L}=\left(L, \leq_{L}\right)$ be a complete lattice that is a chain with at least four elements. For any involutive negator $\mathfrak{N}$ on $\left(I(L), \leq_{t}\right)$ it holds that $r\left(\mathfrak{N}\left(\left[x_{1}, x_{2}\right]\right)\right)=r\left(\mathfrak{N}\left(\left[x_{1}, 1_{\mathcal{L}}\right]\right)\right)$ and $l\left(\mathfrak{N}\left(\left[x_{1}, x_{2}\right]\right)\right)=l\left(\mathfrak{N}\left(\left[0_{\mathcal{L}}, x_{2}\right]\right)\right)$.

Proof: We prove that $r(\mathfrak{N}(x))=r\left(\mathfrak{N}\left(\left[x_{1}, 1_{\mathcal{L}}\right]\right)\right)$, for all $x=\left[x_{1}, x_{2}\right]$ in $I(L)$ (the proof that $l(\mathfrak{N}(x))=l\left(\mathfrak{N}\left(\left[0_{\mathcal{L}}, x_{2}\right]\right)\right)$, for all $x$ in $I(L)$, is similar). When $x_{2}=1_{\mathcal{L}}$, the claim trivially holds, so assume that $x_{2} \quad<_{L} 1_{\mathcal{L}}$, and that $r(\mathfrak{N}(x)) \neq r\left(\mathfrak{N}\left(\left[x_{1}, 1_{\mathcal{L}}\right]\right)\right)$. Then, since $\mathfrak{N}$ is decreasing, we have that $r(\mathfrak{N}(x))>_{L} r\left(\mathfrak{N}\left(\left[x_{1}, 1_{\mathcal{L}}\right]\right)\right)$. Note also that, by
Corollary 6-B, $l\left(\mathfrak{N}\left(\left[x_{1}, 1_{\mathcal{L}}\right]\right)\right)=0_{\mathcal{L}}$. We discuss the following cases.

- If $l(\mathfrak{N}(x))=0_{\mathcal{L}}$, then from Corollary 6-B it follows that $x_{2}=r(\mathfrak{N}(\mathfrak{N}(x)))=1_{\mathcal{L}}$, which is a contradiction.

- If $\left.l(\mathfrak{N}(x)) \in] 0_{\mathcal{L}}, r(\mathfrak{N}(x))\right]$, then let

$$
\begin{aligned}
b & =\left[\min \left(r\left(\mathfrak{N}\left(\left[x_{1}, 1_{\mathcal{L}}\right]\right)\right), l(\mathfrak{N}(x))\right), r\left(\mathfrak{N}\left(\left[x_{1}, 1_{\mathcal{L}}\right]\right)\right)\right] \\
c & =\left[0_{\mathcal{L}}, r(\mathfrak{N}(x))\right] .
\end{aligned}
$$

From $x_{2}<_{L} \quad 1_{\mathcal{L}}$ it follows that $\left[x_{1}, 1_{\mathcal{L}}\right] \neq\left[1_{\mathcal{L}}, 1_{\mathcal{L}}\right]$, so $\mathfrak{N}\left(\left[x_{1}, 1_{\mathcal{L}}\right]\right) \neq\left[0_{\mathcal{L}}, 0_{\mathcal{L}}\right]$. Since from Corollary 6-B it follows that $l\left(\mathfrak{N}\left(\left[x_{1}, 1_{\mathcal{L}}\right]\right)\right)=0_{\mathcal{L}}$, we obtain that $r\left(\mathfrak{N}\left(\left[x_{1}, 1_{\mathcal{L}}\right]\right)\right) \quad>_{L} \quad 0_{\mathcal{L}}$. Hence, $l(b) \quad>_{L} l(c)$. Since from the assumptions about $\mathfrak{N}(x)$ it follows that $r(b)<_{L} r(c)$, we find that $b$ and $c$ are $\leq_{t}$-incomparable. It can be easily verified that $\mathfrak{N}\left(\left[x_{1}, 1_{\mathcal{L}}\right]\right) \leq_{t} b \leq_{t} \mathfrak{N}(x)$ and $\mathfrak{N}\left(\left[x_{1}, 1_{\mathcal{L}}\right]\right) \quad \leq_{t} \quad c \quad \leq_{t} \quad \mathfrak{N}(x)$. Since $\mathfrak{N}$ is decreasing, we obtain that $x \leq_{t} \mathfrak{N}(b) \leq_{t}\left[x_{1}, 1_{\mathcal{L}}\right]$ and $x \leq_{t} \mathfrak{N}(c) \leq_{t}\left[x_{1}, 1_{\mathcal{L}}\right]$. Thus, $l(\mathfrak{N}(b))=l(\mathfrak{N}(c))=x_{1}$, so $\mathfrak{N}(b)$ and $\mathfrak{N}(c)$ are $\leq_{t}$-comparable. Since $\mathfrak{N}$ is decreasing and involutive, it follows that $b$ and $c$ are $\leq_{t}$-comparable, which is a contradiction.

Corollary 6-D: Let $\mathcal{L}=\left(L, \leq_{L}\right)$ be a complete lattice that is a chain with at least four elements. For any involutive negator $\mathfrak{N}$ on $\left(I(L), \leq_{t}\right)$ it holds that

$$
\begin{aligned}
r\left(\mathfrak{N}\left(\left[x_{1}, x_{1}\right]\right)\right) & =r\left(\mathfrak{N}\left(\left[x_{1}, x_{2}\right]\right)\right)=r\left(\mathfrak{N}\left(\left[x_{1}, 1_{\mathcal{L}}\right]\right)\right) \quad \text { and } \\
l\left(\mathfrak{N}\left(\left[x_{2}, x_{2}\right]\right)\right) & =l\left(\mathfrak{N}\left(\left[x_{1}, x_{2}\right]\right)\right)=l\left(\mathfrak{N}\left(\left[0_{\mathcal{L}}, x_{2}\right]\right)\right)
\end{aligned}
$$

for all $\left[x_{1}, x_{2}\right]$ in $I(L)$.

Lemma 6-E: Let $\mathcal{L}=\left(L, \leq_{L}\right)$ be a complete lattice that is a chain with at least four elements. For any involutive negator $\mathfrak{N}$ on $\left(I(L), \leq_{t}\right)$ it holds that $\mathfrak{N}(D) \subseteq D$.

Proof: Obviously, $\mathfrak{N}\left(\left[0_{\mathcal{L}}, 0_{\mathcal{L}}\right]\right)=\left[1_{\mathcal{L}}, 1_{\mathcal{L}}\right] \in D$ and $\mathfrak{N}\left(\left[1_{\mathcal{L}}, 1_{\mathcal{L}}\right]\right)=\left[0_{\mathcal{L}}, 0_{\mathcal{L}}\right] \in D$. Assume that there exists an $x_{1}$ in $L \backslash\left\{0_{\mathcal{L}}, 1_{\mathcal{L}}\right\}$ such that $\mathfrak{N}\left(\left[x_{1}, x_{1}\right]\right)=\left[y_{1}, y_{2}\right]$, with $y_{1}, y_{2}$ in $L$ and $y_{1}<_{L} \quad y_{2}$. From Corollary 6-D it follows that $r\left(\mathfrak{N}\left(\left[y_{1}, y_{1}\right]\right)\right)=r\left(\mathfrak{N}\left(\left[y_{1}, y_{2}\right]\right)\right)=r\left(\left[x_{1}, x_{1}\right]\right)=x_{1}$, using the fact that $\mathfrak{N}$ is involutive. Furthermore, since $\mathfrak{N}$ is a decreasing bijection, $\mathfrak{N}\left(\left[y_{1}, y_{1}\right]\right)>_{t} \mathfrak{N}\left(\left[y_{1}, y_{2}\right]\right)=\left[x_{1}, x_{1}\right]$, so $l\left(\mathfrak{N}\left(\left[y_{1}, y_{1}\right]\right)\right)>_{L} l\left(\left[x_{1}, x_{1}\right]\right)=x_{1}=r\left(\mathfrak{N}\left(\left[y_{1}, y_{1}\right]\right)\right)$, which is a contradiction.

Lemma 6-F: Let $\mathcal{L}=\left(L, \leq_{L}\right)$ be a complete lattice that is a chain with at least four elements and let $\mathfrak{N}$ be a negator on $\left(I(L), \leq_{t}\right)$. Then $\mathfrak{N}$ is involutive if and only if there exists an involutive negator $\mathcal{N}$ on $\left(L, \leq_{L}\right)$ such that, for all $\left[x_{1}, x_{2}\right]$ in $I(L)$

$$
\mathfrak{N}\left(\left[x_{1}, x_{2}\right]\right)=\left[\mathcal{N}\left(x_{2}\right), \mathcal{N}\left(x_{1}\right)\right] .
$$

Proof: Assume first that $\mathfrak{N}$ is an involutive negator on $\left(I(L), \leq_{t}\right)$. Define the mappings $\mathcal{N}_{1}: L \rightarrow L$ and $\mathcal{N}_{2}: L \rightarrow L$ as follows:

$$
\begin{aligned}
& \mathcal{N}_{1}\left(x_{1}\right)=r\left(\mathfrak{N}\left(\left[x_{1}, 1_{\mathcal{L}}\right]\right)\right) \\
& \mathcal{N}_{2}\left(x_{2}\right)=l\left(\mathfrak{N}\left(\left[0_{\mathcal{L}}, x_{2}\right]\right)\right) .
\end{aligned}
$$

Then, from Lemma 6-A it follows that $\mathcal{N}_{1}\left(0_{\mathcal{L}}\right)=1_{\mathcal{L}}$. Obviously, $\mathcal{N}_{1}\left(1_{\mathcal{L}}\right)=0_{\mathcal{L}}$. Since $\mathfrak{N}$ is decreasing, $\mathcal{N}_{1}$ is decreasing. 
Hence, $\mathcal{N}_{1}$ is a negator on $\left(L, \leq_{L}\right)$. Similarly, $\mathcal{N}_{2}$ is a negator on $\left(L, \leq_{L}\right)$.

From Corollary 6-D it follows that

$$
\begin{aligned}
l(\mathfrak{N}(x)) & =l\left(\mathfrak{N}\left(\left[0_{\mathcal{L}}, x_{2}\right]\right)\right)=\mathcal{N}_{2}\left(x_{2}\right) \quad \text { and } \\
r(\mathcal{N}(x)) & =r\left(\mathfrak{N}\left(\left[x_{1}, 1_{\mathcal{L}}\right]\right)\right)=\mathcal{N}_{1}\left(x_{1}\right)
\end{aligned}
$$

for all $x=\left[x_{1}, x_{2}\right]$ in $I(L)$. Since from Lemma 6-E it follows that $\mathfrak{N}\left(\left[x_{1}, x_{1}\right]\right)=\left[\mathcal{N}_{2}\left(x_{1}\right), \mathcal{N}_{1}\left(x_{1}\right)\right]$ is an element of $D$, for all $x_{1}$ in $L$, we obtain that $\mathcal{N}_{1}=\mathcal{N}_{2}$.

Let from now on $\mathcal{N}=\mathcal{N}_{1}=\mathcal{N}_{2}$. From Corollary 6-B it follows, for all $x_{1}, x_{2}$ in $L$

$$
\begin{aligned}
& \mathfrak{N}\left(\left[x_{1}, 1_{\mathcal{L}}\right]\right)=\left[0_{\mathcal{L}}, r\left(\mathfrak{N}\left(\left[x_{1}, 1_{\mathcal{L}}\right]\right)\right)\right]=\left[0_{\mathcal{L}}, \mathcal{N}\left(x_{1}\right)\right] \\
& \mathfrak{N}\left(\left[0_{\mathcal{L}}, x_{2}\right]\right)=\left[l\left(\mathfrak{N}\left(\left[0_{\mathcal{L}}, x_{2}\right]\right)\right), 1_{\mathcal{L}}\right]=\left[\mathcal{N}\left(x_{2}\right), 1_{\mathcal{L}}\right] .
\end{aligned}
$$

Since $\mathfrak{N}$ is involutive, we obtain $\left[x_{1}, 1_{\mathcal{L}}\right]=\mathfrak{N}\left(\mathfrak{N}\left(\left[x_{1}, 1_{\mathcal{L}}\right]\right)\right)=$ $\mathfrak{N}\left(\left[0_{\mathcal{L}}, \mathcal{N}\left(x_{1}\right)\right]\right)=\left[\mathcal{N}\left(\mathcal{N}\left(x_{1}\right)\right), 1_{\mathcal{L}}\right]$, for all $x_{1}$ in $L$. Hence, $\mathcal{N}$ is involutive.

Assume conversely that $\mathcal{N}$ is an involutive negator on $\left(L, \leq_{L}\right)$ and define the mapping $\mathfrak{N}: I(L) \rightarrow I(L)$ by, for all $x=$ $\left[x_{1}, x_{2}\right]$ in $I(L)$

$$
\mathfrak{N}(x)=\left[\mathcal{N}\left(x_{2}\right), \mathcal{N}\left(x_{1}\right)\right] .
$$

Then from the fact that $\mathcal{N}$ is a negator on $\left(L, \leq_{L}\right)$ it easily follows that $\mathfrak{N}\left(\left[0_{\mathcal{L}}, 0_{\mathcal{L}}\right]\right)=\left[1_{\mathcal{L}}, 1_{\mathcal{L}}\right]$ and $\mathfrak{N}\left(\left[1_{\mathcal{L}}, 1_{\mathcal{L}}\right]\right)=\left[0_{\mathcal{L}}, 0_{\mathcal{L}}\right]$. Since $\mathcal{N}$ is decreasing, $\mathfrak{N}$ is decreasing. So $\mathfrak{N}$ is a negator on $\left(I(L), \leq_{t}\right)$. Since $\mathcal{N}$ is involutive, we obtain that $\mathfrak{N}(\mathfrak{N}(x))=$ $\mathfrak{N}\left(\left[\mathcal{N}\left(x_{2}\right), \mathcal{N}\left(x_{1}\right)\right]\right)=\left[\mathcal{N}\left(\mathcal{N}\left(x_{1}\right)\right), \mathcal{N}\left(\mathcal{N}\left(x_{2}\right)\right)\right]=x$, for all $x$ in $I(L)$. Hence, $\mathfrak{N}$ is involutive.

This completes the Proof of Lemma 6-F and Proposition 6.

Note that Proposition 6 is not true unless the chain $\mathcal{L}$ has at least four elements. To see this, consider the following example.

Example 3: Consider a mapping $\mathcal{N}$ on $\left(I\left(L_{3}\right), \leq_{t}\right)$, defined as follows:

$$
\mathcal{N}\left(\left[x_{1}, x_{2}\right]\right)= \begin{cases}{\left[\frac{1}{2}, \frac{1}{2}\right],} & \text { if }\left[x_{1}, x_{2}\right]=[0,1] \\ {[0,1],} & \text { if }\left[x_{1}, x_{2}\right]=\left[\frac{1}{2}, \frac{1}{2}\right] \\ {\left[1-x_{2}, 1-x_{1}\right],} & \text { otherwise }\end{cases}
$$

It is easy to check that this is an involutive negator on $\left(I\left(L_{3}\right)\right.$, $\leq_{t}$ ), which is not of the form of (3) (thus, it is not generated as described in Proposition 6).

In [36] it is shown that there does not exist a Kleene negator on $\left(I([0,1]), \leq_{t}\right)$. Note, however, that as the following examples show, this does not hold in general for any triangle.

Example 4: The operation $\mathcal{N}$, defined by $\mathcal{N}([0,0])=[1,1]$, $\mathcal{N}([1,1])=[0,0]$ and $\mathcal{N}([0,1])=[0,1]$ is a Kleene negator on $\left(I\left(L_{2}\right), \leq_{t}\right)$.

Example 5: Consider a mapping $\mathcal{N}$ on $\left(I\left(L_{3}\right), \leq_{t}\right)$, defined by $\mathcal{N}\left(\left[x_{1}, x_{2}\right]\right)=\left[1-x_{2}, 1-x_{1}\right]$. This is an involutive negator, but it does not satisfy Kleene's condition, since, e.g.,

$$
\begin{aligned}
{[0,1] \wedge \mathcal{N}([0,1]) } & =[0,1] \not \underline{L}_{t}\left[\frac{1}{2}, \frac{1}{2}\right] \\
& =\left[\frac{1}{2}, \frac{1}{2}\right] \vee \mathcal{N}\left(\left[\frac{1}{2}, \frac{1}{2}\right]\right) .
\end{aligned}
$$

Note that by a slight modification of this definition we get the involutive negator of Example 3, which is a Kleene negator on $\left(I\left(L_{3}\right), \leq_{t}\right)$.
In general, we have the following result:

Proposition 7: Let $\mathcal{L}=\left(L, \leq_{L}\right)$ be a complete lattice that is a chain with at least four elements. Then there does not exist a Kleene negator on $\left(I(L), \leq_{t}\right)$.

Proof: Let $\mathcal{N}$ be an involutive negator on $\left(I(L), \leq_{t}\right)$. By Lemma 6-A, $\mathcal{N}\left(\left[0_{\mathcal{L}}, 1_{\mathcal{L}}\right]\right)=\left[0_{\mathcal{L}}, 1_{\mathcal{L}}\right]$. Since $L$ contains more than three elements, there exists an $\left.x_{1} \in\right] 0_{\mathcal{L}}, 1_{\mathcal{L}}$ [ for which $\left[x_{1}, x_{1}\right]$ is incomparable to $\left[0_{\mathcal{L}}, 1_{\mathcal{L}}\right]$ w.r.t. $\leq_{t}$. Furthermore, $\mathcal{N}\left(\left[x_{1}, x_{1}\right]\right)$ is incomparable to $\left[0_{\mathcal{L}}, 1_{\mathcal{L}}\right]$, since otherwise, if $\mathcal{N}\left(\left[x_{1}, x_{1}\right]\right) \leq_{t}\left[0_{\mathcal{L}}, 1_{\mathcal{L}}\right]$, then from Corollary 6-B it would follow that $\left[x_{1}, x_{1}\right] \geq_{t}\left[0_{\mathcal{L}}, 1_{\mathcal{L}}\right]$, which is a contradiction. Similarly, the assumption $\mathcal{N}\left(\left[x_{1}, x_{1}\right]\right) \geq_{t}\left[0_{\mathcal{L}}, 1_{\mathcal{L}}\right]$ leads to a contradiction. Therefore, $r\left(\mathcal{N}\left(\left[x_{1}, x_{1}\right]\right)\right)<_{L} 1_{\mathcal{L}}$.

Now, since $\mathcal{L}$ is a chain, $x \vee_{L} y=1_{\mathcal{L}}$ iff $\left(x=1_{\mathcal{L}}\right.$ or $\left.y=1_{\mathcal{L}}\right)$. Thus, $r\left(\left[x_{1}, x_{1}\right] \vee \mathcal{N}\left(\left[x_{1}, x_{1}\right]\right)\right)=x_{1} \vee_{L} r\left(\mathcal{N}\left(\left[x_{1}, x_{1}\right]\right)\right)<1_{\mathcal{L}}$. It follows that $\left[0_{\mathcal{L}}, 1_{\mathcal{L}}\right] \wedge \mathcal{N}\left(\left[0_{\mathcal{L}}, 1_{\mathcal{L}}\right]\right)=\left[0_{\mathcal{L}}, 1_{\mathcal{L}}\right] \not_{t}\left[x_{1}, x_{1}\right] \vee$ $\mathcal{N}\left(\left[x_{1}, x_{1}\right]\right)$, and so $\mathcal{N}$ is not a Kleene negator on $\left(I(L), \leq_{t}\right)$.

\section{B. Conjunction and Disjunction}

Definition 12: A triangular norm (a t-norm, for short) on a lattice $\mathcal{L}=\left(L, \leq_{L}\right)$ is a mapping $\mathcal{T}: L \times L \rightarrow L$ that is $\leq_{L}$-increasing in both arguments, commutative, associative, and satisfies, for every $x$ in $L, \mathcal{T}\left(1_{\mathcal{L}}, x\right)=x$.

Definition 13: A triangular conorm (a $t$-conorm, for short) on a lattice $\mathcal{L}=\left(L, \leq_{L}\right)$ is a mapping $\mathcal{S}: L \times L \rightarrow L$ that is $\leq_{L}$-increasing in both arguments, commutative, associative, and satisfies, for every $x$ in $L, \mathcal{S}\left(0_{\mathcal{L}}, x\right)=x$.

Given a prebilattice $\mathcal{B}=\left(B, \leq_{t}, \leq_{k}\right)$, its $\leq_{t}$-meet $\wedge$ and $\leq_{k}$-meet $\otimes$ are clearly t-norms on $\left(B, \leq_{t}\right)$ and $\left(B, \leq_{k}\right)$, respectively. Also, the $\leq_{t}$-join $\vee$ and the $\leq_{k}$-join $\oplus$ of $\mathcal{B}$ are t-conorms on $\left(B, \leq_{t}\right)$, and $\left(B, \leq_{k}\right)$, respectively. This implies that for a complete lattice $\mathcal{L}=(L, \leq)$ with a meet $\wedge_{L}$ and a join $\vee_{L}$, the following operations are t-norms on $\left(L^{2}, \leq_{t}\right)$ and $\left(L^{2}, \leq_{k}\right)$, respectively

$$
\begin{aligned}
& \mathcal{T}_{\leq_{t}}\left(\left(x_{1}, x_{2}\right),\left(y_{1}, y_{2}\right)\right)=\left(x_{1} \wedge_{L} y_{1}, x_{2} \vee_{L} y_{2}\right) \\
& \mathcal{T}_{\leq_{k}}\left(\left(x_{1}, x_{2}\right),\left(y_{1}, y_{2}\right)\right)=\left(x_{1} \wedge_{L} y_{1}, x_{2} \wedge_{L} y_{2}\right) .
\end{aligned}
$$

Similarly, the operations below are, respectively, t-conorms on $\left(L^{2}, \leq_{t}\right)$ and $\left(L^{2}, \leq_{k}\right)$

$$
\begin{aligned}
& \mathcal{S}_{\leq_{t}}\left(\left(x_{1}, x_{2}\right),\left(y_{1}, y_{2}\right)\right)=\left(x_{1} \vee_{L} y_{1}, x_{2} \wedge_{L} y_{2}\right) \\
& \mathcal{S}_{\leq_{k}}\left(\left(x_{1}, x_{2}\right),\left(y_{1}, y_{2}\right)\right)=\left(x_{1} \vee_{L} y_{1}, x_{2} \vee_{L} y_{2}\right) .
\end{aligned}
$$

Clearly, $\mathcal{T}_{\leq_{t}}$ is the $\leq_{t}$-greatest t-norm on $\left(L^{2}, \leq_{t}\right)$ and $\mathcal{T}_{\leq_{k}}$ is the $\leq_{k}$-greatest $\mathrm{t}$-norm on $\left(L^{2}, \leq_{k}\right)$. Also, it is easy to see that $\mathcal{S}_{\leq_{t}}$ and $\mathcal{S}_{\leq_{k}}$ are, respectively, the $\leq_{t}$-smallest t-conorm on $\left(L^{2}, \leq_{t}\right)$ and the $\leq_{k}$-smallest t-conorm on $\left(L^{2}, \leq_{k}\right)$. Interestingly, as the following proposition shows, the $\leq_{k}$-extreme $t$-(co)norms are definable by the $\leq_{t}$-extreme $t$-(co)norms and the other way around (see also [37]).

Proposition 8: In every square $\mathcal{L}^{2}$

1)

$$
\begin{aligned}
\mathcal{T}_{\leq_{k}}\left(\left(x_{1}, x_{2}\right),\left(y_{1}, y_{2}\right)\right) & =\mathcal{S}_{\leq_{t}}\left(\mathcal { S } _ { \leq _ { t } } \left(\mathcal{T}_{\leq_{t}}\left(\left(x_{1}, x_{2}\right),\left(0_{\mathcal{L}}, 0_{\mathcal{L}}\right)\right),\right.\right. \\
& \left.\mathcal{T}_{\leq_{t}}\left(\left(y_{1}, y_{2}\right),\left(0_{\mathcal{L}}, 0_{\mathcal{L}}\right)\right)\right), \\
& \left.\mathcal{T}_{\leq_{t}}\left(\left(x_{1}, x_{2}\right),\left(y_{1}, y_{2}\right)\right)\right)
\end{aligned}
$$


2)

$$
\begin{aligned}
\mathcal{S}_{\leq_{k}}\left(\left(x_{1}, x_{2}\right),\left(y_{1}, y_{2}\right)\right) & \\
= & \mathcal{S}_{\leq_{t}}\left(\mathcal { S } _ { \leq _ { t } } \left(\mathcal{T}_{\leq_{t}}\left(\left(x_{1}, x_{2}\right),\left(1_{\mathcal{L}}, 1_{\mathcal{L}}\right)\right),\right.\right. \\
& \left.\mathcal{T}_{\leq_{t}}\left(\left(y_{1}, y_{2}\right),\left(1_{\mathcal{L}}, 1_{\mathcal{L}}\right)\right)\right) \\
& \left.\mathcal{T}_{\leq_{t}}\left(\left(x_{1}, x_{2}\right),\left(y_{1}, y_{2}\right)\right)\right)
\end{aligned}
$$

3)

$$
\begin{aligned}
\mathcal{T}_{\leq_{t}}\left(\left(x_{1}, x_{2}\right),\left(y_{1}, y_{2}\right)\right) & \\
= & \mathcal{S}_{\leq_{k}}\left(\mathcal { S } _ { \leq _ { k } } \left(\mathcal{T}_{\leq_{k}}\left(\left(x_{1}, x_{2}\right),\left(0_{\mathcal{L}}, 1_{\mathcal{L}}\right)\right),\right.\right. \\
& \left.\mathcal{T}_{\leq_{k}}\left(\left(y_{1}, y_{2}\right),\left(0_{\mathcal{L}}, 1_{\mathcal{L}}\right)\right)\right), \\
& \left.\mathcal{T}_{\leq_{k}}\left(\left(x_{1}, x_{2}\right),\left(y_{1}, y_{2}\right)\right)\right)
\end{aligned}
$$

4)

$$
\begin{aligned}
& \mathcal{T}_{\leq_{t}}\left(\left(x_{1}, x_{2}\right),\left(y_{1}, y_{2}\right)\right) \\
&= \mathcal{S}_{\leq_{k}}\left(\mathcal { S } _ { \leq _ { k } } \left(\mathcal{T}_{\leq_{k}}\left(\left(x_{1}, x_{2}\right),\left(1_{\mathcal{L}}, 0_{\mathcal{L}}\right)\right),\right.\right. \\
&\left.\mathcal{T}_{\leq_{k}}\left(\left(y_{1}, y_{2}\right),\left(1_{\mathcal{L}}, 0_{\mathcal{L}}\right)\right)\right), \\
&\left.\mathcal{T}_{\leq_{k}}\left(\left(x_{1}, x_{2}\right),\left(y_{1}, y_{2}\right)\right)\right) .
\end{aligned}
$$

Proof: We show only part 1); the proof of the other parts is similar.

$$
\begin{aligned}
& \mathcal{S}_{\leq_{t}}\left(\mathcal{S}_{\leq_{t}}\left(\mathcal{T}_{\leq_{t}}\left(\left(x_{1}, x_{2}\right),\left(0_{\mathcal{L}}, 0_{\mathcal{L}}\right)\right), \mathcal{T}_{\leq_{t}}\left(\left(y_{1}, y_{2}\right),\left(0_{\mathcal{L}}, 0_{\mathcal{L}}\right)\right)\right),\right. \\
&\left.\quad \mathcal{T}_{\leq_{t}}\left(\left(x_{1}, x_{2}\right),\left(y_{1}, y_{2}\right)\right)\right) \\
&=\mathcal{S}_{\leq_{t}}\left(\mathcal{S}_{\leq_{t}}\left(\left(x_{1} \wedge_{L} 0_{\mathcal{L}}, x_{2} \vee_{L} 0_{\mathcal{L}}\right),\left(y_{1} \wedge_{L} 0_{\mathcal{L}}, y_{2} \vee_{L} 0_{\mathcal{L}}\right)\right),\right. \\
&\left.\left(x_{1} \wedge_{L} y_{1}, x_{2} \vee_{L} y_{2}\right)\right) \\
&= \mathcal{S}_{\leq_{t}}\left(\mathcal{S}_{\leq_{t}}\left(\left(0_{\mathcal{L}}, x_{2}\right),\left(0_{\mathcal{L}}, y_{2}\right)\right),\left(x_{1} \wedge_{L} y_{1}, x_{2} \vee_{L} y_{2}\right)\right) \\
&= \mathcal{S}_{\leq_{t}}\left(\left(0_{\mathcal{L}}, x_{2} \wedge_{L} y_{2}\right),\left(x_{1} \wedge_{L} y_{1}, x_{2} \vee_{L} y_{2}\right)\right) \\
&=\left(x_{1} \wedge_{L} y_{1}, x_{2} \wedge_{L} y_{2} \wedge_{L}\left(x_{2} \vee_{L} y_{2}\right)\right) .
\end{aligned}
$$

By the absorption law, $y_{2}=y_{2} \wedge_{L}\left(x_{2} \vee_{L} y_{2}\right)$, so the element in the last line above is equal to $\left(x_{1} \wedge_{L} y_{1}, x_{2} \wedge_{L} y_{2}\right)$, which is $\mathcal{T}_{\leq_{k}}\left(\left(x_{1}, x_{2}\right),\left(y_{1}, y_{2}\right)\right)$.

The definition of $\mathcal{T}_{<_{t}}, \mathcal{S}_{<_{t}}, \mathcal{T}_{<_{k}}$, and $\mathcal{S}_{<_{k}}$ is an example of an effective way of generating t-(co)norms on (substructures of) squares $\mathcal{L}^{2}$ by taking advantage of existing connectives on the underlying lattice $\mathcal{L}$. Intuitively, this amounts to a kind of divideand-conquer strategy, where conjunction and disjunction on $\mathcal{L}^{2}$ are split up into simpler operations on $\mathcal{L}$. This leads us to define the notion of $\mathcal{L}$-representability. ${ }^{4}$

Definition 14: Let $\mathcal{L}=\left(L, \leq_{L}\right)$ be a complete lattice. A t-norm $\mathfrak{T}$ on $\left(L^{2}, \leq_{t}\right)$ (respectively, a t-conorm $\mathfrak{S}$ ) is called $\mathcal{L}$ representable, if there exist a t-norm $\mathcal{T}$ and a t-conorm $\mathcal{S}$ on $\mathcal{L}$ (respectively, a t-conorm $\mathcal{S}^{\prime}$ and a t-norm $\mathcal{T}^{\prime}$ on $\mathcal{L}$ ) such that, for every $\left(x_{1}, x_{2}\right),\left(y_{1}, y_{2}\right)$ in $L^{2}$,

$$
\begin{aligned}
& \mathfrak{T}\left(\left(x_{1}, x_{2}\right),\left(y_{1}, y_{2}\right)\right)=\left(\mathcal{T}\left(x_{1}, y_{1}\right), \mathcal{S}\left(x_{2}, y_{2}\right)\right) \\
& \mathfrak{S}\left(\left(x_{1}, x_{2}\right),\left(y_{1}, y_{2}\right)\right)=\left(\mathcal{S}^{\prime}\left(x_{1}, y_{1}\right), \mathcal{T}^{\prime}\left(x_{2}, y_{2}\right)\right)
\end{aligned}
$$

$\mathcal{T}$ and $\mathcal{S}$ (respectively, $\mathcal{S}^{\prime}$ and $\mathcal{T}^{\prime}$ ) are called the representants of $\mathfrak{T}$ (respectively, $\mathfrak{S}$ ).

Analogously, $\mathcal{L}$-representable t-(co)norms on $\left(L^{2}, \leq_{k}\right)$ can be defined in the obvious way. In the sequel, if the identity of the lattice is clear from the context, we shall simply be speaking about representable $\mathrm{t}-(\mathrm{co})$ norms.

This definition allows a straightforward construction of t-(co)norms by operations that meet Definitions 12 and 13; it suffices to take any t-norm $\mathcal{T}$ and t-conorm $\mathcal{S}$ on $\mathcal{L}$, and to use them as representants in (4) and (5). The converse, however, is

\footnotetext{
${ }^{4}$ This definition extends the notion of $t$-representability, introduced in [38]. To avoid confusion with the $\leq_{t}$-ordering of a bilattice, we will not use the latter terminology in this paper.
}

not true; not any t-(co)norm on $\mathcal{L}^{2}$ can be obtained by a representation. For instance, in [39] it is shown that the mapping $\mathcal{T}:[0,1]^{2} \rightarrow[0,1]$, given by

$$
\begin{aligned}
\mathcal{T}\left(\left(x_{1}, x_{2}\right),\left(y_{1}, y_{2}\right)\right) & \\
& =\left(\min \left(x_{1}, y_{1}, \max \left(x_{2}, y_{2}\right)\right), \min \left(x_{2}, y_{2}\right)\right)
\end{aligned}
$$

is indeed a t-norm on $\left([0,1]^{2}, \leq_{k}\right)$, but clearly it is not $\mathcal{L}$-representable, since its first component also depends on $x_{2}$ and $y_{2}$.

Proposition 9: Let $\mathfrak{T}$ (respectively, $\mathfrak{S}$ ) be an $\mathcal{L}$-representable t-norm (respectively, t-conorm) on $\left(L^{2}, \leq_{t}\right)$. Then $\mathfrak{T}$ (respectively, S) is monotonic with respect to both $\leq_{t}$ and $\leq_{k}$.

The same property holds for $\mathcal{L}$-representable $\mathrm{t}$-(co)norms with respect to $\left(L^{2}, \leq_{k}\right)$.

Proof: Let $\mathcal{T}$ be a t-norm and $\mathcal{S}$ a t-conorm on $\left(L, \leq_{L}\right)$. Consider the $\mathcal{L}$-representable t-norm $\mathfrak{T}$ on $\left(L^{2}, \leq_{t}\right)$, defined by $\mathfrak{T}\left(\left(x_{1}, x_{2}\right),\left(y_{1}, y_{2}\right)\right)=\left(\mathcal{T}\left(x_{1}, y_{1}\right), \mathcal{S}\left(x_{2}, y_{2}\right)\right)$, and suppose that $\left(x_{1}, x_{2}\right) \leq_{k}\left(x_{1}^{\prime}, x_{2}^{\prime}\right)$. Then $x_{1} \quad \leq_{L} \quad x_{1}^{\prime}$ and $x_{2} \leq_{L} x_{2}^{\prime}$, and so, for every $y$ in $L, \mathcal{T}\left(x_{1}, y\right) \leq_{L} \mathcal{T}\left(x_{1}^{\prime}, y\right)$ and $\mathcal{S}\left(x_{2}, y\right) \leq_{L} \mathcal{S}\left(x_{2}^{\prime}, y\right)$. This implies that for every $\left(y_{1}, y_{2}\right)$ in $L^{2},\left(\mathcal{T}\left(x_{1}, y_{1}\right), \mathcal{S}\left(x_{2}, y_{2}\right)\right) \leq_{k}\left(\mathcal{T}\left(x_{1}^{\prime}, y_{1}\right), \mathcal{S}\left(x_{2}^{\prime}, y_{2}\right)\right)$, and therefore $\mathfrak{T}\left(\left(x_{1}, x_{2}\right),\left(y_{1}, y_{2}\right)\right) \leq_{k} \mathfrak{T}\left(\left(x_{1}^{\prime}, x_{2}^{\prime}\right),\left(y_{1}, y_{2}\right)\right)$. The proof of the other cases is similar.

When a t-norm is not $\mathcal{L}$-representable the proposition above is no longer true.

Example 6: Let $\mathcal{T}$ be the t-norm defined in (6). Consider $\left(x_{1}, x_{2}\right)=(0.5,1),\left(x_{1}^{\prime}, x_{2}^{\prime}\right)=(0.5,0)$, and $\left(y_{1}, y_{2}\right)=(1,0)$. Then $\left(x_{1}, x_{2}\right) \leq_{t}\left(x_{1}^{\prime}, x_{2}^{\prime}\right)$, but

$$
\begin{aligned}
\mathcal{T}\left(\left(x_{1}, x_{2}\right),\left(y_{1}, y_{2}\right)\right) & =(\min (0.5,1, \max (1,0)), \min (1,0)) \\
& =(0.5,0) \\
\mathcal{T}\left(\left(x_{1}^{\prime}, x_{2}^{\prime}\right),\left(y_{1}, y_{2}\right)\right) & =(\min (0.5,1, \max (0,0)), \min (0,0)) \\
& =(0,0) .
\end{aligned}
$$

Hence, $\mathcal{T}\left(\left(x_{1}, x_{2}\right),\left(y_{1}, y_{2}\right)\right) \nless_{t} \mathcal{T}\left(\left(x_{1}^{\prime}, x_{2}^{\prime}\right),\left(y_{1}, y_{2}\right)\right)$.

Next we relate t-norms and t-conorms by appropriate negators. A natural way of doing so is to impose de Morgan's laws.

Definition 15: Let $\mathcal{T}$ be a t-norm on $\mathcal{L}$, and let $\mathcal{N}$ be an involutive negator on $\mathcal{L}$. The $\mathcal{N}$-dual of $\mathcal{T}$ is a t-conorm $\mathcal{S}$ on $\mathcal{L}$ defined by

$$
\mathcal{S}(x, y)=\mathcal{N}(\mathcal{T}(\mathcal{N}(x), \mathcal{N}(y)))
$$

The $\mathcal{N}$-dual of a given t-conorm is defined as a t-norm on $\mathcal{L}$ in a similar way.

It is interesting to note that for $\mathcal{L}$-representable $\mathrm{t}$-norms with $\mathcal{N}$-dual representants on, e.g., $\left(L^{2}, \leq_{t}\right)$, the choice of the negator $\sim_{\mathcal{N}}$ or $\neg$ does not affect the identity of the dual t-conorm.

Proposition 10: Suppose that $\mathfrak{T}$ is an $\mathcal{L}$-representable t-norm on $\left(L^{2}, \leq_{t}\right)$ with representants $(\mathcal{T}, \mathcal{S})$, such that $\mathcal{T}$ is the $\mathcal{N}$-dual t-norm of $\mathcal{S}$ and $\mathcal{N}$ is an involutive negator on $\mathcal{L}$. Then the $\left(\sim_{\mathcal{N}}\right)$-dual and the $(\neg)$-dual of $\mathfrak{T}$ are the same.

Proof: For $\left(x_{1}, x_{2}\right),\left(y_{1}, y_{2}\right)$ in $L^{2}$ we have

$$
\begin{aligned}
\neg \mathfrak{T} & \left(\neg\left(x_{1}, x_{2}\right), \neg\left(y_{1}, y_{2}\right)\right) \\
\quad= & \neg\left(\mathcal{T}\left(x_{2}, y_{2}\right), \mathcal{S}\left(x_{1}, y_{1}\right)\right) \\
\quad & =\left(\mathcal{S}\left(x_{1}, y_{1}\right), \mathcal{T}\left(x_{2}, y_{2}\right)\right)
\end{aligned}
$$


and

$$
\begin{array}{rl}
\sim_{\mathcal{N}} & \mathfrak{T}\left(\sim_{\mathcal{N}}\left(x_{1}, x_{2}\right), \sim_{\mathcal{N}}\left(y_{1}, y_{2}\right)\right) \\
\quad= & \sim_{\mathcal{N}}\left(\mathcal{T}\left(\mathcal{N}\left(x_{1}\right), \mathcal{N}\left(y_{1}\right)\right), \mathcal{S}\left(\mathcal{N}\left(x_{2}\right), \mathcal{N}\left(y_{2}\right)\right)\right) \\
& =\left(\mathcal{N}\left(\mathcal{T}\left(\mathcal{N}\left(x_{1}\right), \mathcal{N}\left(y_{1}\right)\right)\right), \mathcal{N}\left(\mathcal{S}\left(\mathcal{N}\left(x_{2}\right), \mathcal{N}\left(y_{2}\right)\right)\right)\right) \\
& =\left(\mathcal{S}\left(x_{1}, y_{1}\right), \mathcal{T}\left(x_{2}, y_{2}\right)\right) .
\end{array}
$$

Thus

$\neg \mathfrak{T}\left(\neg\left(x_{1}, x_{2}\right), \neg\left(y_{1}, y_{2}\right)\right)=\sim_{\mathcal{N}} \mathfrak{T}\left(\sim_{\mathcal{N}}\left(x_{1}, x_{2}\right), \sim_{\mathcal{N}}\left(y_{1}, y_{2}\right)\right)$

A similar discussion as above also applies to the definitions of t-(co)norms on (substructures of) the triangle $\mathcal{I}(\mathcal{L})$, with the caveat that

- there are neither t-norms nor t-conorms on $\left(I(L), \leq_{k}\right)$, and

- in the choice of representants on $\mathcal{L}$ it must be assured that the resulting composite operation always yields an element of $I(L)$.

\section{Implication}

Definition 16: An implicator on a lattice $\mathcal{L}=\left(L, \leq_{L}\right)$ is a mapping $\mathcal{I}: L \times L \rightarrow L$ that is $\leq_{L}$-increasing in its first component and $\leq_{L}$-decreasing in its second component, such that

$$
\begin{array}{ll}
\mathcal{I}\left(0_{\mathcal{L}}, 0_{\mathcal{L}}\right)=1_{\mathcal{L}} & \mathcal{I}\left(1_{\mathcal{L}}, 0_{\mathcal{L}}\right)=0_{\mathcal{L}} \\
\mathcal{I}\left(0_{\mathcal{L}}, 1_{\mathcal{L}}\right)=1_{\mathcal{L}} & \mathcal{I}\left(1_{\mathcal{L}}, 1_{\mathcal{L}}\right)=1_{\mathcal{L}} .
\end{array}
$$

Given a t-norm $\mathcal{T}$ and an implicator $\mathcal{I}$ on $\mathcal{L}$, it is usual to require the soundness of fuzzy modus ponens, i.e., for $a, b, x, y$ in $L$, it should hold that

$$
\text { if } a \leq_{L} x \quad \text { and } \quad b \leq_{L} \mathcal{I}(x, y), \quad \text { then } \quad \mathcal{T}(a, b) \leq_{L} y .
$$

In particular, therefore, if for some $z$ in $L, z \leq_{L} \mathcal{I}(x, y)$ then $\mathcal{T}(x, z) \leq_{L} y$. On the other hand, to allow $\mathcal{I}(x, y)$ to be as large as possible, one would like to require the converse, that $\mathcal{T}(x, z) \leq_{L} y$ implies $z \leq_{L} \mathcal{I}(x, y)$. Eventually, then

$$
\mathcal{T}(x, z) \leq_{L} y \Leftrightarrow z \leq_{L} \mathcal{I}(x, y)
$$

a condition which is known also as the residuation principle, and which leads to the following class of implicators.

Definition 7: Let $\mathcal{T}$ be a t-norm on $\mathcal{L}$. An $\mathcal{R}$-implicator $\mathcal{I}_{\mathcal{T}}$ (the residuum of $\mathcal{T}$ ) is defined, for every $x, y$ in $L$, by $\mathcal{I}_{\mathcal{T}}(x, y)=\sup \left\{z \in L \mid \mathcal{T}(x, z) \leq_{L} y\right\}$.

Note 2: In [40, Property 2.48], it was shown that (7) holds if and only if $\mathcal{I}=\mathcal{I}_{\mathcal{T}}$ and if $\mathcal{T}$ satisfies, for any set $\left\{x_{i}\right\}_{i \in I}$ in $L$, $\mathcal{T}\left(\sup _{i \in I} x_{i}, y\right)=\sup _{i \in I} \mathcal{T}\left(x_{i}, y\right)$.

In the sequel, we will say that a t-norm $\mathcal{T}$ satisfies the residuation principle if (7) holds for $\mathcal{T}$ together with $\mathcal{I}=\mathcal{I}_{\mathcal{T}}$. The following proposition will be important for our further exposition.

Proposition 11: Let $\mathcal{L}=\left(L, \leq_{L}\right)$ be a complete lattice and $\mathcal{T}$ a t-norm on $\left(L^{2}, \leq_{t}\right)$ satisfying the residuation principle. Then $\mathcal{T}$ is $\mathcal{L}$-representable.
Proof: In [41] it was proven that if $\mathcal{T}$ satisfies $\mathcal{T}(x \vee y$, $z)=\mathcal{T}(x, z) \vee \mathcal{T}(y, z)$, then $\mathcal{T}$ is $\mathcal{L}$-representable. Now, the claim immediately follows from Note 2 .

Another definition of a family of implicators is motivated by the classical definition of the material implication $x \rightarrow y$ as $\neg x \vee y$.

Definition 18: Let $\mathcal{S}$ be a t-conorm and $\mathcal{N}$ a negator on $\mathcal{L}$. The $\mathcal{S}$-implicator $\mathcal{I}_{\mathcal{S}, \mathcal{N}}($ generated by $\mathcal{S}$ and $\mathcal{N}$ ) is defined for every $x, y$ in $L$ by $\mathcal{I}_{\mathcal{S}, \mathcal{N}}(x, y)=\mathcal{S}(\mathcal{N}(x), y)$.

It is easy to verify that each $\mathcal{R}$-implicator and each $\mathcal{S}$-implicator is in particular an implicator. Moreover, these definitions reveal that very often implicators are linked to "simpler" connectives. Also, we can exploit the classical equivalence between the formulas $x \rightarrow f$ and $\neg x$, to define the following special kind of negator on $\mathcal{L}$.

Definition 19: Let $\mathcal{L}=(L, \leq)$ be a complete lattice with an implicator $\mathcal{I}$. The induced negator of $\mathcal{I}$ is a mapping $\mathcal{N}_{\mathcal{I}}$, defined for every $x$ in $L$ by $\mathcal{N}_{\mathcal{I}}(x)=\mathcal{I}\left(x, 0_{\mathcal{L}}\right)$.

Examples of all the above operations on bilattice-based squares and triangles are, thus, easy to generate using the constructs introduced in the previous sections. As an illustration, and in view of its importance for the sequel, the following proposition derives an explicit representation of $\mathcal{R}$-implicators of $\mathcal{L}$-representable t-norms on $\left(L^{2}, \leq_{t}\right)$.

Proposition 12: Let $\mathcal{L}=\left(L, \leq_{L}\right)$ be a complete lattice and let $\mathfrak{T}$ be an $\mathcal{L}$-representable t-norm on $\left(L^{2}, \leq_{t}\right)$ with representants $\mathcal{T}$ and $\mathcal{S}$, where $\mathcal{S}$ is the $\mathcal{N}$-dual t-conorm of a t-norm $\mathcal{T}^{\prime}$ for some involutive negator $\mathcal{N}$. Then, for every $\left(x_{1}, x_{2}\right),\left(y_{1}, y_{2}\right)$ in $L^{2}$

$\mathcal{I}_{\mathfrak{T}}\left(\left(x_{1}, x_{2}\right),\left(y_{1}, y_{2}\right)\right)$

$$
=\left(\mathcal{I}_{\mathcal{T}}\left(x_{1}, y_{1}\right), \mathcal{N}\left(\mathcal{I}_{\mathcal{T}^{\prime}}\left(\mathcal{N}\left(x_{2}\right), \mathcal{N}\left(y_{2}\right)\right)\right)\right) .
$$

Proof: Indeed

$$
\begin{aligned}
\mathcal{I}_{\mathfrak{T}} & \left(\left(x_{1}, x_{2}\right),\left(y_{1}, y_{2}\right)\right) \\
= & \sup \left\{\left(\gamma_{1}, \gamma_{2}\right) \in L^{2} \mid \mathfrak{T}\left(\left(x_{1}, x_{2}\right),\left(\gamma_{1}, \gamma_{2}\right)\right) \leq_{t} y\right\} \\
= & \sup \left\{\left(\gamma_{1}, \gamma_{2}\right) \in L^{2} \mid \mathcal{T}\left(x_{1}, \gamma_{1}\right) \leq_{L} y_{1}\right. \\
\quad & \left.\quad \operatorname{sad} \quad \mathcal{S}\left(x_{2}, \gamma_{2}\right) \geq_{L} y_{2}\right\} \\
= & \left(\sup \left\{\gamma_{1} \in L \mid \mathcal{T}\left(x_{1}, \gamma_{1}\right) \leq_{L} y_{1}\right\},\right. \\
& \left.\quad \inf \left\{\gamma_{2} \in L \mid \mathcal{S}\left(x_{2}, \gamma_{2}\right) \geq_{L} y_{2}\right\}\right) \\
= & \left(\mathcal{I}_{\mathcal{T}}\left(x_{1}, y_{1}\right), \inf \left\{\gamma_{2} \in L \mid \mathcal{N}\left(\mathcal{T}^{\prime}\left(\mathcal{N}\left(x_{2}\right), \mathcal{N}\left(\gamma_{2}\right)\right)\right)\right.\right. \\
= & \left(\mathcal{I}_{\mathcal{T}}\left(x_{1}, y_{1}\right), \inf \left\{y_{2} \in L \mid \mathcal{T}^{\prime}\left(\mathcal{N}\left(x_{2}\right), \mathcal{N}\left(\gamma_{2}\right)\right)\right.\right. \\
\quad & \left.\left({ }_{L} \mathcal{N}\left(y_{2}\right)\right\}\right) \\
& \left(\mathcal{I}_{\mathcal{T}}\left(x_{1}, y_{1}\right), \mathcal{N}\left(\operatorname { s u p } \left\{\gamma_{2} \in L \mid \mathcal{T}^{\prime}\left(\mathcal{N}\left(x_{2}\right), \gamma_{2}\right)\right.\right.\right. \\
= & \left(\mathcal{I}_{\mathcal{T}}\left(x_{1}, y_{1}\right), \mathcal{N}\left(\mathcal{I}_{\mathcal{T}^{\prime}}\left(\mathcal{N}\left(x_{2}\right), \mathcal{N}\left(y_{2}\right)\right)\right)\right) .
\end{aligned}
$$

The question of implicator classification, i.e., which particular instance to use in which case, has received considerable attention in the literature. A comprehensive account for the situation in the lattice $\left(I([0,1]), \leq_{t}\right)$ appears in $[25]$ and $\left([0,1]^{2}, \leq_{t}\right)$ is examined in [24] and [36]. 
Here, in the context of bilattices, it is in particular interesting to investigate the light that implicators shed on the choice of the "right" negator. To that aim, first observe that in classical bilattices, the most "natural" $\mathcal{R}$-implicator, i.e., the one based on $\wedge$, coincides with an $\mathcal{S}$-implicator on condition that the associated negator is chosen as $\sim$ (i.e., the combination $-\neg$ ):

Proposition 13: Let $\mathcal{B}=\left(B, \leq_{t}, \leq_{k}, \neg\right)$ be a classical bilattice with conflation - . Then $\mathcal{I}_{\wedge}=\mathcal{I}_{(\vee, \sim)}$, and so $\mathcal{I}_{\wedge}$ is both an $\mathcal{S}$-implicator and an $\mathcal{R}$-implicator on $\left(B, \leq_{t}\right)$.

Proof: First, as $\wedge$ is a t-norm, $\vee$ is a t-conorm, and $\mathcal{N}(x)=$ $-\neg x$ is a negator of $\left(B, \leq_{t}\right), \mathcal{I}_{\wedge}$ is indeed an $\mathcal{R}$-implicator and $\mathcal{I}_{(\vee,-\neg)}$ is an $\mathcal{S}$-implicator. Second, by Definition 2 and Proposition 1 , in every classical bilattice it holds that $a \wedge-\neg a=f$ for every $a$ in $B$. Now, for every $a, b$ in $B$

$$
\begin{aligned}
a \wedge b \leq_{t} b & \Rightarrow f \vee(a \wedge b) \leq_{t} b \\
& \Rightarrow(a \wedge-\neg a) \vee(a \wedge b) \leq_{t} b \\
& \Rightarrow a \wedge(-\neg a \vee b) \leq_{t} b .
\end{aligned}
$$

Thus, $\mathcal{I}_{(\vee,-\neg)} \leq_{t} \mathcal{I}_{\wedge}$. On the other hand, by the definition of $\mathcal{I}_{\wedge}$

$$
\begin{aligned}
a & \wedge \mathcal{I}_{\wedge}(a, b) \leq_{t} b \\
& \Rightarrow-\neg a \vee\left(a \wedge \mathcal{I}_{\wedge}(a, b)\right) \leq_{t}-\neg a \vee b \\
& \Rightarrow(-\neg a \vee a) \wedge\left(-\neg a \vee \mathcal{I}_{\wedge}(a, b)\right) \leq_{t}-\neg a \vee b \\
& \Rightarrow t \wedge\left(-\neg a \vee \mathcal{I}_{\wedge}(a, b)\right) \leq_{t}-\neg a \vee b \\
& \Rightarrow-\neg a \vee \mathcal{I}_{\wedge}(a, b) \leq_{t}-\neg a \vee b .
\end{aligned}
$$

Thus, also $\mathcal{I}_{\wedge} \leq_{t} \mathcal{I}_{(\vee,-\neg)}$, and so $\mathcal{I}_{\wedge}=\mathcal{I}_{(\vee, \sim)}$.

Proposition 13 substantiates the claim, hinted at by Definition 2 , that in classical bilattices the combination " $\neg \neg$ " is the one that really plays the role of Boolean negation, and the formulas $x \vee-\neg x$ are the analogies of classical tautologies. An alternative proof of the above result can be given in terms of MV-algebras, of which Boolean algebras are a special case; the following definition is not a reproduction of the original, lengthy one given in [42], but is rather a minimal characterization in terms of required properties (see, e.g., [43]).

Definition 20: Let $\mathcal{L}=\left(L, \leq_{L}\right)$ be a complete lattice, and let $\mathcal{T}$ be a t-norm on $\mathcal{L}$ that satisfies the residuation principle (7) and such that, for every $x, y$ in $L$

$$
\mathcal{I}_{\mathcal{T}}\left(\mathcal{I}_{\mathcal{T}}(x, y), y\right)=\mathcal{I}_{\mathcal{T}}\left(\mathcal{I}_{\mathcal{T}}(y, x), x\right)=x \vee_{L} y
$$

Then $\left(L, \leq_{L}, \mathcal{T}\right)$ is called an $M V$-algebra.

Proposition 13 then follows from the following observation, along with the fact that any Boolean algebra $\left(B, \wedge_{B}, \vee_{B}, \neg_{B}\right)$ is an MV-algebra in which $\mathcal{T}$ coincides with $\wedge_{B}$ (see e.g., [43]).

Proposition 14: In an MV-algebra $\left(L, \leq_{L}, \mathcal{T}\right)$, the mappings $\mathcal{I}_{\mathcal{T}}$ and $\mathcal{I}_{\mathcal{S}, \mathcal{N}}$, where $\mathcal{N}=\mathcal{N}_{\mathcal{I}_{\mathcal{T}}}$ and $\mathcal{S}$ is the $\mathcal{N}$-dual of $\mathcal{T}$, are identical.

Now we investigate what happens in squares that correspond to nonclassical bilattices. In this context it is easy to show that the converse of Proposition 14 is not true in general, that is: coinciding $\mathcal{R}$ - and $\mathcal{S}$-implicators would not necessarily lead to an MV-algebra. To see this consider, e.g., the lattice $([0,1], \leq)$.
It is easy to verify that the nilpotent minimum, defined for every $x, y$ in $[0,1]$ by

$$
\mathcal{T}(x, y)= \begin{cases}\min (x, y), & \text { if } x+y>1 \\ 0, & \text { otherwise }\end{cases}
$$

is a t-norm on this lattice. Now, for $\mathcal{N}=\mathcal{N}_{\mathcal{I}_{\mathcal{T}}}$ and the $\mathcal{N}$-dual $\mathcal{S}$ of $\mathcal{T}$, we have that

$$
\mathcal{I}_{\mathcal{T}}(x, y)=\mathcal{I}_{\mathcal{S}, \mathcal{N}}(x, y)= \begin{cases}1, & \text { if } x \leq y \\ \max (1-x, y), & \text { otherwise }\end{cases}
$$

Still, this implicator does not satisfy (8) of Definition 20, and so $([0,1], \leq, \mathcal{T})$ is not an MV-algebra.

The last example can also be extended to $\left([0,1]^{2}, \leq_{t}\right)$ with a representable t-norm $\mathfrak{T}$ on it, whose representants are the above nilpotent minimum $\mathcal{T}$ and the t-conorm $\mathcal{S}$, which is the dual of $\mathcal{T}$ with respect to the standard negation on $[0,1]$. By Proposition 11 , the residual implicator of $\mathfrak{T}$ is given by

$$
\begin{aligned}
\mathcal{I}_{\mathfrak{T}}\left(\left(x_{1}, x_{2}\right),\left(y_{1}, y_{2}\right)\right) & \\
= & \left(\mathcal{I}_{\mathcal{T}}\left(x_{1}, y_{1}\right), 1-\mathcal{I}_{\mathcal{T}}\left(1-x_{2}, 1-y_{2}\right)\right)
\end{aligned}
$$

and the induced negator of $\mathcal{I}_{\mathfrak{T}}, \mathcal{N}=\mathcal{N}_{\mathcal{I}_{\mathfrak{T}}}$, is given by

$$
\begin{aligned}
\mathcal{N}\left(\left(x_{1}, x_{2}\right)\right) & =\mathcal{I}_{\mathfrak{T}}\left(\left(x_{1}, x_{2}\right),(0,1)\right) \\
& =\left(\mathcal{I}_{\mathcal{T}}\left(x_{1}, 0\right), 1-\mathcal{I}_{\mathcal{T}}\left(1-x_{2}, 1-x_{1}\right)\right) \\
& =\left(1-x_{1}, 1-x_{2}\right) .
\end{aligned}
$$

The associated $\mathcal{S}$-implicator is, thus

$$
\begin{aligned}
\mathcal{I}_{\mathfrak{S}, \mathcal{N}} & \left(\left(x_{1}, x_{2}\right),\left(y_{1}, y_{2}\right)\right) \\
& =\mathfrak{S}\left(\mathcal{N}\left(x_{1}, x_{2}\right),\left(y_{1}, y_{2}\right)\right) \\
& =\left(\mathcal{S}\left(1-x_{1}, y_{1}\right), \mathcal{T}\left(1-x_{2}, y_{2}\right)\right) \\
& =\left(\mathcal{I}_{\mathcal{T}}\left(x_{1}, y_{1}\right), 1-\mathcal{S}\left(x_{2}, 1-y_{2}\right)\right) \\
& =\left(\mathcal{I}_{\mathcal{T}}\left(x_{1}, y_{1}\right), 1-\mathcal{I}_{\mathcal{T}}\left(1-x_{2}, 1-y_{2}\right)\right. \\
& =\mathcal{I}_{\mathfrak{T}}\left(\left(x_{1}, x_{2}\right),\left(y_{1}, y_{2}\right)\right) .
\end{aligned}
$$

So the $\mathcal{R}$ - and $\mathcal{S}$-implicators that are associated with $\mathfrak{T}$ coincide, but by the same argument as in the previous example, $\mathcal{T}$ does not generate an MV-algebra on $\left([0,1]^{2}, \leq_{t}\right)$.

A necessary and sufficient condition for having coinciding $\mathcal{R}$ - and $\mathcal{S}$-implicators in arbitrary squares (not only those that correspond to classical bilattices; cf. Propositions 13 and 14), is given in the following proposition.

Proposition 15: Let $\mathcal{L}=\left(L, \leq_{L}\right)$ be a complete lattice, and let $\mathfrak{T}$ be a t-norm on $\left(L^{2}, \leq_{t}\right)$. Then the $\mathcal{R}$-implicator $\mathfrak{I}_{\mathfrak{T}}$ of $\mathfrak{T}$ on $\left(L^{2}, \leq_{t}\right)$ is equal to the $\mathcal{S}$-implicator $\mathfrak{I}_{\mathfrak{S}, \mathfrak{N}}$ generated by an involutive negator $\mathfrak{N}$ on $\left(L^{2}, \leq_{t}\right)$ and the $\mathfrak{N}$-dual t-conorm $\mathfrak{S}$ of $\mathfrak{T}$, if and only if there exist t-norms $\mathcal{T}$ and $\mathcal{T}^{\prime}$ on $\mathcal{L}$ for which $\mathcal{I}_{\mathcal{T}}=\mathcal{I}_{\mathcal{S}, \mathcal{N}}$ and $\mathcal{I}_{\mathcal{I}^{\prime}}=\mathcal{I}_{\mathcal{S}^{\prime}, \mathcal{N}^{\prime}}$, where $\mathcal{N}$ and $\mathcal{N}^{\prime}$ are involutive negators on $\mathcal{L}$ and where $\mathcal{S}$ (respectively, $\mathcal{S}^{\prime}$ ) is the $\mathcal{N}$-dual (respectively $\mathcal{N}^{\prime}$-dual) t-conorm of $\mathcal{T}$ (respectively, $\mathcal{T}^{\prime}$ ) such that $\mathfrak{T}$ is $\mathcal{L}$-representable with representants $\mathcal{T}$ and $\mathcal{S}^{\prime}$.

Proof: For the proof we need the following lemma:

Lemma 15-A: Let $\mathcal{L}^{\prime}=\left(L^{\prime}, \leq{ }_{L^{\prime}}\right)$ be a complete lattice. If the $\mathcal{R}$-implicator $\mathcal{I}_{\mathcal{T}}$ of a t-norm $\mathcal{T}$ on $\mathcal{L}^{\prime}$ is equal to the $\mathcal{S}$-implicator $\mathcal{I}_{\mathcal{S}, \mathcal{N}}$ generated by an involutive negator $\mathcal{N}$ on $\mathcal{L}^{\prime}$ and the $\mathcal{N}$-dual t-conorm $\mathcal{S}$ of $\mathcal{T}$, then $\mathcal{T}$ satisfies the residuation principle. 
Proof: If for a t-norm $\mathcal{T}$ on $\mathcal{L}^{\prime}$ it holds that $\mathcal{I}_{\mathcal{T}}=\mathcal{I}_{\mathcal{S}, \mathcal{N}}$, then by the definition of residual implicator we obtain that, for all $x, y \in L^{\prime}$

$$
\begin{aligned}
\mathcal{N}(\mathcal{T}(x, \mathcal{N}(y))) & =\mathcal{S}(\mathcal{N}(x), y) \\
& =\sup \left\{z \in L^{\prime} \mid \mathcal{T}(x, z) \leq_{L^{\prime}} y\right\} .
\end{aligned}
$$

Let $x, y, z \in L^{\prime}$. If $\mathcal{T}(x, z) \leq_{L^{\prime}} y$, then $z \in\{z \in$ $\left.L^{\prime} \mid \mathcal{T}(x, z) \leq_{L^{\prime}} y\right\}$, so $z \leq_{L^{\prime}} \mathcal{I}_{\mathcal{T}}(x, z)=\mathcal{N}(\mathcal{T}(x, \mathcal{N}(y)))$. Since $\mathcal{N}$ is involutive, then

$$
\mathcal{T}(x, z) \leq_{L^{\prime}} y \Rightarrow \mathcal{T}(x, \mathcal{N}(y)) \leq_{L^{\prime}} \mathcal{N}(z) .
$$

If we replace in (9) $z$ by $\mathcal{N}(y)$ and $y$ by $\mathcal{N}(z)$, we have the following:

$$
\mathcal{T}(x, \mathcal{N}(y)) \leq_{L^{\prime}} \mathcal{N}(z) \Rightarrow \mathcal{T}(x, z) \leq_{L^{\prime}} y .
$$

Combining (9) and (10), we get, for all $x, y, z \in L^{\prime}$,

$$
\mathcal{T}(x, z) \leq{ }_{L^{\prime}} y \Leftrightarrow z \leq_{L^{\prime}} \mathcal{N}(\mathcal{T}(x, \mathcal{N}(y)))=\mathcal{I}_{\mathcal{T}}(x, y)
$$

and so $\mathcal{T}$ satisfies the residuation principle.

Now we can show Proposition 15.

$(\Rightarrow)$ Let $\mathcal{L}^{\prime}=\left(L^{2}, \leq_{t}\right)$. If $\mathfrak{T}$ is a t-norm on $\left(L^{2}, \leq_{t}\right)$ for which $\mathfrak{I}_{\mathfrak{T}}=\mathfrak{I}_{\mathfrak{S}, \mathfrak{N}}$, for some involutive negator $\mathfrak{N}$ on $\left(L^{2}, \leq_{t}\right)$, then $\mathfrak{T}$ satisfies the residuation principle by Lemma 15 -A. Note that it also follows that $\mathfrak{N}=\mathfrak{N}_{\mathfrak{I}_{\tau}}$.

From Propositions 11 and 12 , it follows that $\mathfrak{T}$ is $\mathcal{L}$-representable (we call the representants $\mathcal{T}$ and $\mathcal{S}^{\prime}$ ), and that, for all $x, y \in L^{2}$

$$
\mathfrak{I}_{\mathfrak{T}}(x, y)=\left(\mathcal{I}_{\mathcal{T}}\left(x_{1}, y_{1}\right), \mathcal{N}\left(\mathcal{I}_{\mathcal{T}^{\prime}}\left(\mathcal{N}\left(x_{2}\right), \mathcal{N}\left(y_{2}\right)\right)\right)\right)
$$

where $\mathcal{T}^{\prime}$ denotes the $\mathcal{N}$-dual t-norm of $\mathcal{S}^{\prime}$, for an arbitrary involutive negator $\mathcal{N}$ on $\mathcal{L}$. The negator $\mathfrak{N}_{\mathfrak{I}_{\mathfrak{T}}}$ induced by $\mathfrak{I}_{\mathfrak{T}}$ is given, for all $x \in L^{2}$, by

$$
\mathfrak{N}_{\mathfrak{I}_{\mathfrak{T}}}(x)=\left(\mathcal{N}_{\mathcal{I}_{\mathcal{T}}}\left(x_{1}\right), \mathcal{N}\left(\mathcal{N}_{\mathcal{I}_{\mathcal{T}}}\left(\mathcal{N}\left(x_{2}\right)\right)\right)\right) .
$$

Consider now the $\mathcal{S}$-implicator $\mathfrak{I}_{\mathfrak{S}, \mathfrak{N}}$ generated by $\mathfrak{N}$ and the $\mathfrak{N}$-dual $\mathfrak{S}$ of $\mathfrak{T}$. Using the fact that $\mathfrak{N}=\mathfrak{N}_{\mathfrak{I}_{\mathfrak{T}}}$, the implicator $\mathfrak{I}_{\mathfrak{S}, \mathfrak{N}}$ is defined, for all $x, y \in L^{2}$, by

$$
\begin{aligned}
& \mathfrak{I}_{\mathfrak{S}, \mathfrak{N}}(x, y) \\
& =\mathfrak{S}(\mathfrak{N}(x), y) \\
& =\mathfrak{N}(\mathfrak{T}(x, \mathfrak{N}(y))) \\
& =\left(\mathcal{N}_{\mathcal{I}_{\mathcal{T}}}\left(\mathcal{T}\left(x_{1}, \mathcal{N}_{\mathcal{I}_{\mathcal{T}}}\left(y_{1}\right)\right)\right),\right. \\
& \mathcal{N}\left(\mathcal{N}_{\mathcal{I}_{\mathcal{T}^{\prime}}}\left(\mathcal{N}\left(\mathcal{S}^{\prime}\left(x_{2}, \mathcal{N}\left(\mathcal{N}_{\mathcal{I}_{\mathcal{T}^{\prime}}}\left(\mathcal{N}\left(y_{2}\right)\right)\right)\right)\right)\right)\right) \\
& =\left(\mathcal{N}_{\mathcal{I}_{\mathcal{T}}}\left(\mathcal{T}\left(x_{1}, \mathcal{N}_{\mathcal{I}_{\mathcal{T}}}\left(y_{1}\right)\right)\right)\right. \text {, } \\
& \left.\mathcal{N}\left(\mathcal{N}_{\mathcal{I}_{\mathcal{T}^{\prime}}}\left(\mathcal{T}^{\prime}\left(\mathcal{N}\left(x_{2}\right), \mathcal{N}_{\mathcal{I}_{\mathcal{T}^{\prime}}}\left(\mathcal{N}\left(y_{2}\right)\right)\right)\right)\right)\right) \\
& =\left(\mathcal{S}\left(\mathcal{N}_{\mathcal{I}_{\mathcal{T}}}\left(x_{1}\right), y_{1}\right), \mathcal{N}\left(\mathcal{S}^{\prime \prime}\left(\mathcal{N}_{\mathcal{I}_{\mathcal{T}^{\prime}}}\left(\mathcal{N}\left(x_{2}\right)\right), \mathcal{N}\left(y_{2}\right)\right)\right)\right) \\
& =\left(\mathcal{I}_{\mathcal{S}, \mathcal{N}_{\mathcal{I}_{\mathcal{T}}}}\left(x_{1}, y_{1}\right), \mathcal{N}\left(\mathcal{I}_{\mathcal{S}^{\prime \prime}, \mathcal{N}_{\mathcal{I}^{\prime}}}\left(\mathcal{N}\left(x_{2}\right), \mathcal{N}\left(y_{2}\right)\right)\right)\right)
\end{aligned}
$$

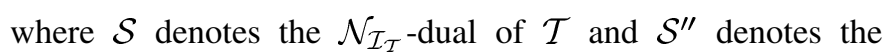
$\mathcal{N}_{\mathcal{I}_{\mathcal{T}}}$-dual of $\mathcal{T}^{\prime}$. Combining this with (11), we obtain that $\mathcal{I}_{\mathcal{T}}=\mathcal{I}_{\mathcal{S}, \mathcal{N}_{\mathcal{I}_{\mathcal{T}}}}$ and $\mathcal{I}_{\mathcal{T}^{\prime}}=\mathcal{I}_{\mathcal{S}^{\prime \prime}, \mathcal{N}_{\mathcal{I}_{\mathcal{I}}}}$. Now, since the arguments above hold for every involutive negator $\mathcal{N}$ on $\mathcal{L}$, we can choose $\mathcal{N}=\mathcal{N}_{\mathcal{I}_{\mathcal{T}^{\prime}}}$. Then $\mathcal{S}^{\prime}=\mathcal{S}^{\prime \prime}$ and we are done.
$(\Leftarrow)$ Assume that $\mathcal{T}$ and $\mathcal{T}^{\prime}$ are t-norms on $\mathcal{L}$ for which $\mathcal{I}_{\mathcal{T}}=\mathcal{I}_{\mathcal{S}, \mathcal{N}}$ and $\mathcal{I}_{\mathcal{T}^{\prime}}=\mathcal{I}_{\mathcal{S}^{\prime}, \mathcal{N}^{\prime}}$, where $\mathcal{N}$ and $\mathcal{N}^{\prime}$ are involutive negators on $\mathcal{L}$ and where $\mathcal{S}$ is the $\mathcal{N}$-dual of $\mathcal{T}$ and $\mathcal{S}^{\prime}$ is the $\mathcal{N}^{\prime}$-dual of $\mathcal{T}^{\prime}$. Let $\mathfrak{T}$ be the $\mathcal{L}$-representable t-norm on $\left(L^{2}, \leq_{t}\right)$ with representants $\mathcal{T}$ and $\mathcal{S}^{\prime}$. From Proposition 12 again, it follows that the $\mathcal{R}$-implicator $\mathfrak{I}_{\mathfrak{T}}$ of $\mathfrak{T}$ is given, for all $x, y \in L^{2}$, by

$$
\mathfrak{I}_{\mathfrak{T}}(x, y)=\left(\mathcal{I}_{\mathcal{T}}\left(x_{1}, y_{1}\right), \mathcal{N}^{\prime}\left(\mathcal{I}_{\mathcal{T}^{\prime}}\left(\mathcal{N}^{\prime}\left(x_{2}\right), \mathcal{N}^{\prime}\left(y_{2}\right)\right)\right)\right) .
$$

Using the fact that $\mathcal{I}_{\mathcal{T}}=\mathcal{I}_{\mathcal{S}, \mathcal{N}}$ and $\mathcal{I}_{\mathcal{T}^{\prime}}=\mathcal{I}_{\mathcal{S}^{\prime}, \mathcal{N}^{\prime}}$, we obtain, for all $x, y \in L^{2}$,

$$
\begin{aligned}
\mathfrak{I}_{\mathfrak{T}} & (x, y) \\
& =\left(\mathcal{I}_{\mathcal{S}, \mathcal{N}}\left(x_{1}, y_{1}\right), \mathcal{N}^{\prime}\left(\mathcal{I}_{\mathcal{S}^{\prime}, \mathcal{N}^{\prime}}\left(\mathcal{N}^{\prime}\left(x_{2}\right), \mathcal{N}^{\prime}\left(y_{2}\right)\right)\right)\right) \\
& =\left(\mathcal{S}\left(\mathcal{N}\left(x_{1}\right), y_{1}\right), \mathcal{N}^{\prime}\left(\mathcal{S}^{\prime}\left(x_{2}, \mathcal{N}^{\prime}\left(y_{2}\right)\right)\right)\right) \\
& =\left(\mathcal{S}\left(\mathcal{N}\left(x_{1}\right), y_{1}\right), \mathcal{T}^{\prime}\left(\mathcal{N}^{\prime}\left(x_{2}\right), y_{2}\right)\right) \\
& =\mathfrak{S}(\mathfrak{N}(x), y)
\end{aligned}
$$

where $\mathfrak{N}$ is the involutive negator on $\left(L^{2}, \leq_{t}\right)$ defined, for all $x \in L^{2}$, by $\mathfrak{N}(x)=\left(\mathcal{N}\left(x_{1}\right), \mathcal{N}^{\prime}\left(x_{2}\right)\right)$, and where $\mathfrak{S}$ is the $\mathfrak{N}$-dual t-conorm of $\mathfrak{T}$. Hence, $\mathfrak{I}_{\mathfrak{T}}=\mathfrak{I}_{\mathfrak{S}, \mathfrak{N}}$.

The following example advances some interesting observations about the choice of a proper negator for a given square, and how this affects the relationships between the corresponding implicators as well as the existence of associated MV-algebras.

Example 7: Consider $\left([0,1]^{2}, \leq_{t}\right)$. In [36] it was shown that the mapping $\mathfrak{T}$, defined by

$$
\begin{aligned}
\mathfrak{T} & \left(\left(x_{1}, x_{2}\right),\left(y_{1}, y_{2}\right)\right) \\
\quad & =\left(\mathcal{T}\left(x_{1}, y_{1}\right), \mathcal{S}\left(x_{2}, y_{2}\right)\right) \\
& =\left(\max \left(0, x_{1}+y_{1}-1\right), \min \left(1, x_{2}+y_{2}\right)\right)
\end{aligned}
$$

is a representable t-norm that satisfies the residuation principle, and, moreover, that $\mathcal{I}_{\mathfrak{T}}=\mathcal{I}_{\mathfrak{S}, \sim_{\mathcal{N}}}$, where $\mathcal{N}(x)=1-x$ and $\mathfrak{S}$ is the $\sim_{\mathcal{N}}$-dual of $\mathfrak{T}$. On the other hand, $\mathcal{I}_{\mathfrak{T}} \neq \mathcal{I}_{\mathfrak{S}, \neg}$. This is another clear hint in favour of the negation-conflation combination $\sim_{\mathcal{N}}$.

Moreover, $\left([0,1]^{2}, \leq_{t}, \mathfrak{T}\right)$ is an MV-algebra; below we show that property (8) in Definition 20 holds. Indeed, note that in our case $\mathcal{I}_{\mathfrak{T}}$ is given by

$$
\begin{array}{r}
\mathcal{I}_{\mathfrak{T}}\left(\left(x_{1}, x_{2}\right),\left(y_{1}, y_{2}\right)\right) \\
=\left(\min \left(1, y_{1}+1-x_{1}\right), \max \left(0, y_{2}-x_{2}\right)\right) .
\end{array}
$$

Now, for $x=\left(x_{1}, x_{2}\right)$ and $y=\left(y_{1}, y_{2}\right)$ in $[0,1]^{2}$ we have the following:

$$
\begin{aligned}
\mathcal{I}_{\mathfrak{T}} & \left.\mathcal{I}_{\mathfrak{T}}(x, y), y\right) \\
= & \mathcal{I}_{\mathfrak{T}}\left(\left(\min \left(1, y_{1}+1-x_{1}\right), \max \left(0, y_{2}-x_{2}\right)\right),\left(y_{1}, y_{2}\right)\right) \\
= & \left(\min \left(1, y_{1}+1-\min \left(1, y_{1}+1-x_{1}\right)\right)\right. \\
& \left.\max \left(0, y_{2}-\max \left(0, y_{2}-x_{2}\right)\right)\right) \\
= & \left(\min \left(1, \max \left(y_{1}+1-1, y_{1}+1-y_{1}-1+x_{1}\right)\right)\right. \\
& \left.\max \left(0, \min \left(y_{2}, y_{2}-y_{2}+x_{2}\right)\right)\right) \\
= & \left(\max \left(y_{1}, x_{1}\right), \min \left(y_{2}, x_{2}\right)\right) \\
= & \left(x_{1}, x_{2}\right) \vee\left(y_{1}, y_{2}\right) \\
= & x \vee y .
\end{aligned}
$$


Note that for the above example it holds that $\left(L, \leq_{L}, \mathcal{T}\right)$ is an MV-algebra, and that $\mathcal{S}$ is the $\mathcal{N}_{\mathcal{I}_{\mathcal{T}}}$-dual t-conorm of $\mathcal{T}$. So, the structure $\left(L^{2}, \leq_{t}, \mathfrak{T}\right)$ "contains," by way of its representants, two underlying MV-algebras. The following proposition clarifies the general picture.

Proposition 16: Let $\mathcal{L}=\left(L, \leq_{L}\right)$ be a complete lattice, and let $\mathfrak{T}$ be a t-norm on $\left(L^{2}, \leq_{t}\right)$. Then $\left(L^{2}, \leq_{t}, \mathfrak{T}\right)$ is an MV-algebra if and only if there exist t-norms $\mathcal{T}$ and $\mathcal{T}^{\prime}$ on $\mathcal{L}$ such that $\left(L, \leq_{L}, \mathcal{T}\right)$ and $\left(L, \leq_{L}, \mathcal{T}^{\prime}\right)$ are both $\mathrm{MV}$-algebras, and such that $\mathfrak{T}$ is $\mathcal{L}$-representable with representants $\mathcal{T}$ and $\mathcal{S}$, where $\mathcal{S}$ is the $\mathcal{N}$-dual t-conorm of a t-norm $\mathcal{T}^{\prime}$ for some involutive negator $\mathcal{N}$.

Proof: $(\Rightarrow)$ Let $\left(L^{2}, \leq_{t}, \mathfrak{T}\right)$ be an MV-algebra. By Proposition 11, $\mathfrak{T}$ is $\mathcal{L}$-representable, i.e., there exist a t-norm $\mathcal{T}$ and a t-conorm $\mathcal{S}$ on $\mathcal{L}$, such that $\mathfrak{T}\left(\left(x_{1}, x_{2}\right),\left(y_{1}, y_{2}\right)\right)=\left(\mathcal{T}\left(x_{1}, y_{1}\right)\right.$, $\left.\mathcal{S}\left(x_{2}, y_{2}\right)\right)$. Since $\left(L^{2}, \leq_{t}, \mathfrak{T}\right)$ is an MV-algebra, it holds that

$$
\begin{aligned}
\mathfrak{T}\left(\left(x_{1}, x_{2}\right),\left(y_{1}, y_{2}\right)\right) \leq_{t}\left(z_{1}, z_{2}\right) & \\
& \Leftrightarrow\left(x_{1}, x_{2}\right) \leq_{t} \mathcal{I}_{\mathfrak{T}}\left(\left(y_{1}, y_{2}\right),\left(z_{1}, z_{2}\right)\right)
\end{aligned}
$$

or equivalently, by Proposition 12

$$
\begin{aligned}
& \mathcal{T}\left(x_{1}, y_{1}\right) \leq_{L} z_{1} \quad \text { and } \quad \mathcal{S}\left(x_{2}, y_{2}\right) \geq_{L} z_{2} \\
& \Leftrightarrow x_{1} \leq_{L} \mathcal{I}_{\mathcal{T}}\left(y_{1}, z_{1}\right) \quad \text { and } \quad x_{2} \geq_{L} \mathcal{N}\left(\mathcal{I}_{\mathcal{T}^{\prime}}\left(\mathcal{N}\left(y_{2}\right), \mathcal{N}\left(z_{2}\right)\right)\right)
\end{aligned}
$$

where $\mathcal{T}^{\prime}$ is the $\mathcal{N}$-dual t-norm of $\mathcal{S}$ with respect to some negator $\mathcal{N}$. Thus

$$
\begin{aligned}
& \mathcal{T}\left(x_{1}, y_{1}\right) \leq_{L} z_{1} \quad \text { and } \quad \mathcal{T}^{\prime}\left(\mathcal{N}\left(x_{2}\right), \mathcal{N}\left(y_{2}\right)\right) \leq_{L} \mathcal{N}\left(z_{2}\right) \\
& \Leftrightarrow x_{1} \leq_{L} \mathcal{I}_{\mathcal{T}}\left(y_{1}, z_{1}\right) \quad \text { and } \quad \mathcal{N}\left(x_{2}\right) \leq_{L} \mathcal{I}_{\mathcal{T}^{\prime}}\left(\mathcal{N}\left(y_{2}\right), \mathcal{N}\left(z_{2}\right)\right)
\end{aligned}
$$

which means that both $\mathcal{T}$ and $\mathcal{T}^{\prime}$ satisfy the residuation condition. To see that $\mathcal{T}$ and $\mathcal{T}^{\prime}$ also satisfy the condition of (8), note that since $\left(L^{2}, \leq_{t}, \mathfrak{T}\right)$ is an MV-algebra

$$
\mathcal{I}_{\mathfrak{T}}\left(\mathcal{I}_{\mathfrak{T}}\left(\left(x_{1}, x_{2}\right),\left(y_{1}, y_{2}\right)\right),\left(y_{1}, y_{2}\right)\right)=\left(x_{1}, x_{2}\right) \vee\left(y_{1}, y_{2}\right) \text {. }
$$

By Proposition 12, the left-hand side of the last equality is

$$
\begin{aligned}
\mathcal{I}_{\mathfrak{T}} & \left(\mathcal{I}_{\mathfrak{T}}\left(\left(x_{1}, x_{2}\right),\left(y_{1}, y_{2}\right)\right),\left(y_{1}, y_{2}\right)\right) \\
= & \mathcal{I}_{\mathfrak{T}}\left(\left(\mathcal{I}_{\mathcal{T}}\left(x_{1}, y_{1}\right), \mathcal{N}\left(\mathcal{I}_{\mathcal{T}^{\prime}}\left(\mathcal{N}\left(x_{2}\right), \mathcal{N}\left(y_{2}\right)\right)\right)\right),\left(y_{1}, y_{2}\right)\right) \\
= & \left(\mathcal{I}_{\mathcal{T}}\left(\mathcal{I}_{\mathcal{T}}\left(x_{1}, y_{1}\right), y_{1}\right)\right. \\
& \mathcal{N}\left(\mathcal{I}_{\mathcal{T}^{\prime}}\left(\mathcal{I}_{\mathcal{T}^{\prime}}\left(\mathcal{N}\left(x_{2}\right), \mathcal{N}\left(y_{2}\right)\right), \mathcal{N}\left(y_{2}\right)\right)\right)
\end{aligned}
$$

and the right-hand side is given by

$$
\begin{aligned}
& \left(x_{1}, x_{2}\right) \vee_{L}\left(y_{1}, y_{2}\right) \\
& \quad=\left(x_{1} \vee_{L} y_{1}, x_{2} \wedge_{L} y_{2}\right) \\
& \quad=\left(x_{1} \vee_{L} y_{1}, \mathcal{N}\left(\mathcal{N}\left(x_{2}\right) \vee_{L} \mathcal{N}\left(y_{2}\right)\right)\right)
\end{aligned}
$$

Therefore

$\mathcal{I}_{\mathcal{T}}\left(\mathcal{I}_{\mathcal{T}}\left(x_{1}, y_{1}\right), y_{1}\right)=x_{1} \vee_{L} y_{1} \quad$ and

$\mathcal{N}\left(\mathcal{I}_{\mathcal{T}^{\prime}}\left(\mathcal{I}_{\mathcal{T}^{\prime}}\left(\mathcal{N}\left(x_{2}\right), \mathcal{N}\left(y_{2}\right)\right), \mathcal{N}\left(y_{2}\right)\right)=\mathcal{N}\left(\mathcal{N}\left(x_{2}\right) \vee_{L} \mathcal{N}\left(y_{2}\right)\right)\right.$

which means that both $\mathcal{T}$ and $\mathcal{T}^{\prime}$ satisfy (8), and so $\left(L, \leq_{L}, \mathcal{T}\right)$ and $\left(L, \leq_{L}, \mathcal{T}^{\prime}\right)$ are $\mathrm{MV}$-algebras.
$(\Leftarrow)$ We shall show that $\mathfrak{T}$ satisfies the conditions of (7) and (8). To see that the residuation principle holds, note that

$$
\begin{aligned}
& \mathfrak{T}\left(\left(x_{1}, x_{2}\right),\left(y_{1}, y_{2}\right)\right) \leq_{t}\left(z_{1}, z_{2}\right) \\
& \quad \Leftrightarrow \mathcal{T}\left(x_{1}, y_{1}\right) \leq_{L} z_{1} \quad \text { and } \quad \mathcal{S}\left(x_{2}, y_{2}\right) \geq_{L} z_{2} \\
& \quad \Leftrightarrow \mathcal{T}\left(x_{1}, y_{1}\right) \leq_{L} z_{1} \quad \text { and } \quad \mathcal{T}^{\prime}\left(\mathcal{N}\left(x_{2}\right), \mathcal{N}\left(y_{2}\right)\right) \leq_{L} \mathcal{N}\left(z_{2}\right) \\
& \quad \Leftrightarrow x_{1} \leq_{L} \mathcal{I}_{\mathcal{T}}\left(y_{1}, z_{1}\right) \quad \text { and } \quad \mathcal{N}\left(x_{2}\right) \leq_{L} \mathcal{I}_{\mathcal{T}^{\prime}}\left(\mathcal{N}\left(y_{2}\right), \mathcal{N}\left(z_{2}\right)\right) \\
& \quad \Leftrightarrow x_{1} \leq_{L} \mathcal{I}_{\mathcal{T}}\left(y_{1}, z_{1}\right) \quad \text { and } \quad x_{2} \geq_{L} \mathcal{N}\left(\mathcal{I}_{\mathcal{T}^{\prime}}\left(\mathcal{N}\left(y_{2}\right), \mathcal{N}\left(z_{2}\right)\right)\right) \\
& \quad \Leftrightarrow\left(x_{1}, x_{2}\right) \leq_{t}\left(\mathcal{I}_{\mathcal{T}}\left(y_{1}, z_{1}\right), \mathcal{N}\left(\mathcal{I}_{\mathcal{T}^{\prime}}\left(\mathcal{N}\left(y_{2}\right), \mathcal{N}\left(z_{2}\right)\right)\right)\right) \\
& \quad \Leftrightarrow\left(x_{1}, x_{2}\right) \leq_{t} \mathcal{I}_{\mathfrak{T}}\left(\left(y_{1}, y_{2}\right),\left(z_{1}, z_{2}\right)\right) .
\end{aligned}
$$

To see (8), note that

$$
\begin{aligned}
\mathcal{I}_{\mathfrak{T}}\left(\mathcal{I}_{\mathfrak{T}}\right. & \left.\left(\left(x_{1}, x_{2}\right),\left(y_{1}, y_{2}\right)\right),\left(y_{1}, y_{2}\right)\right) \\
= & \mathcal{I}_{\mathfrak{T}}\left(\left(\mathcal{I}_{\mathcal{T}}\left(x_{1}, y_{1}\right), \mathcal{N}\left(\mathcal{I}_{\mathcal{T}^{\prime}}\left(\mathcal{N}\left(x_{2}\right), \mathcal{N}\left(y_{2}\right)\right)\right)\right),\left(y_{1}, y_{2}\right)\right) \\
= & \left(\mathcal{I}_{\mathcal{T}}\left(\mathcal{I}_{\mathcal{T}}\left(x_{1}, y_{1}\right), y_{1}\right),\right. \\
& \mathcal{N}\left(\mathcal{I}_{\mathcal{T}^{\prime}}\left(\mathcal{I}_{\mathcal{T}^{\prime}}\left(\mathcal{N}\left(x_{2}\right), \mathcal{N}\left(y_{2}\right)\right), \mathcal{N}\left(y_{2}\right)\right)\right) \\
& =\left(x_{1} \vee_{L} y_{1}, \mathcal{N}\left(\mathcal{N}\left(x_{2}\right) \vee_{L} \mathcal{N}\left(y_{2}\right)\right)\right) \\
& =\left(x_{1} \vee_{L} y_{1}, x_{2} \wedge_{L} y_{2}\right) \\
& =\left(x_{1}, x_{2}\right) \vee\left(y_{1}, y_{2}\right) .
\end{aligned}
$$

\begin{tabular}{|c|c|c|c|c|c|c|c|}
\hline $\mathcal{T}$ & {$[0,0]$} & {$[1,1]$} & {$[0,1]$} & $\mathcal{I}$ & {$[0,0]$} & {$[1,1]$} & {$[0,1]$} \\
\hline$[0,0]$ & {$[0,0]$} & {$[0,0]$} & {$[0,0]$} & {$[0,0]$} & {$[1,1]$} & {$[1,1]$} & {$[1,1]$} \\
\hline$[1,1]$ & {$[0,0]$} & {$[1,1]$} & {$[0,1]$} & {$[1,1]$} & {$[0,0]$} & {$[1,1]$} & {$[0,1]$} \\
\hline$[0,1]$ & {$[0,0]$} & {$[0,1]$} & {$[0,0]$} & {$[0,1]$} & {$[0,1]$} & {$[1,1]$} & {$[1,1]$} \\
\hline
\end{tabular}

It follows, then, that $\left(L^{2}, \leq_{t}, \mathfrak{T}\right)$ is an MV-algebra.

We turn now to triangles. In these structures the situation is complicated by the fact that there need not exist a Kleene negator on $\left(I(L), \leq_{t}\right)$, while this is a prerequisite of an MV-algebra (see, [44]). ${ }^{5}$ The following example summarizes previous findings when $\mathcal{L}$ is the unit interval (see, e.g., [24]).

Example 8: Consider the triangle $\left(I([0,1]), \leq_{t}\right)$. The mapping $\mathcal{T}$, defined by

$$
\begin{aligned}
\mathcal{T}\left(\left[x_{1}, x_{2}\right],\left[y_{1}, y_{2}\right]\right)= & {\left[\max \left(0, x_{1}+y_{1}-1\right)\right.} \\
& \left.\max \left(0, x_{2}-1+y_{1}, y_{2}-1+x_{1}\right)\right]
\end{aligned}
$$

is a nonrepresentable t-norm that satisfies the residuation principle (7). Moreover, here $\mathcal{I}_{\mathcal{T}}=\mathcal{I}_{\mathcal{S}, \neg}$, where $\mathcal{S}$ is the $(\neg)$-dual of $\mathcal{T}$. However, since there is no Kleene negator on $\left(I([0,1]), \leq_{t}\right)$, the triple $\left(I([0,1]), \leq_{t}, \mathcal{T}\right)$ is not an MV-algebra.

Example 8 shows that the property of having coinciding $\mathcal{R}$ and $\mathcal{S}$-implicators is not unique to MV-algebras. Conversely, one might also wonder if substructures of bilattice-based triangles can ever be MV-algebras; the following example answers this question in the affirmative.

Example 9: Consider the triangle $\mathcal{I}\left(\mathcal{L}_{2}\right)$. As we have seen, the mapping $\mathcal{N}$ defined in Example 4 is a Kleene negator on $\left(I\left(L_{2}\right), \leq_{t}\right)$. Consider the following truth tables that define a t-norm $\mathcal{T}$ and an implicator $\mathcal{I}$ on $\left(I\left(L_{2}\right), \leq_{t}\right)$ :

We have that $\mathcal{I}=\mathcal{I}_{\mathcal{T}}$, the residuation principle is satisfied in this case, and as the following truth table shows, $\left(I\left(L_{2}\right), \leq_{t}, \mathcal{T}\right)$ is an MV-algebra.

5Indeed, if $\left(\mathcal{L}, \leq_{L}, \mathcal{T}\right)$ is an MV-algebra, then $\mathcal{N}_{\mathcal{I}_{\mathcal{T}}}$ is a Kleene negator on $\mathcal{L} ;$ see [44, Theorem 2.31] 


\begin{tabular}{c|c||c|c|c}
$x$ & $y$ & $\mathcal{I}(x, y)$ & $\mathcal{I}(\mathcal{I}(x, y), y)$ & $x \vee y$ \\
\hline$[0,0]$ & {$[0,0]$} & {$[1,1]$} & {$[0,0]$} & {$[0,0]$} \\
{$[0,0]$} & {$[1,1]$} & {$[1,1]$} & {$[1,1]$} & {$[1,1]$} \\
{$[0,0]$} & {$[0,1]$} & {$[1,1]$} & {$[0,1]$} & {$[0,1]$} \\
{$[1,1]$} & {$[0,0]$} & {$[0,0]$} & {$[1,1]$} & {$[1,1]$} \\
{$[1,1]$} & {$[1,1]$} & {$[1,1]$} & {$[1,1]$} & {$[1,1]$} \\
{$[1,1]$} & {$[0,1]$} & {$[0,1]$} & {$[1,1]$} & {$[1,1]$} \\
{$[0,1]$} & {$[0,0]$} & {$[0,1]$} & {$[0,1]$} & {$[0,1]$} \\
{$[0,1]$} & {$[1,1]$} & {$[1,1]$} & {$[1,1]$} & {$[1,1]$} \\
{$[0,1]$} & {$[0,1]$} & {$[1,1]$} & {$[0,1]$} & {$[0,1]$}
\end{tabular}

Now, the following question might be raised: if a Kleene negator exists on $\left(I(L), \leq_{t}\right)$, can a t-norm $\mathcal{T}$ always be found such that $\left(I(L), \leq_{t}, \mathcal{T}\right)$ would be an MV-algebra? The following example gives a negative answer to this question.

Example 10: Suppose that there exists a t-norm $\mathcal{T}$ on the triangle $\mathcal{I}\left(\mathcal{L}_{3}\right)=\left(I\left(L_{3}\right), \leq_{t}\right)$, satisfying (7) and (8). Then $[0,1] \vee[1 / 2,1 / 2]=$ $[1 / 2,1]=\mathcal{I}_{\mathcal{T}}\left(\mathcal{I}_{\mathcal{T}}([0,1],[1 / 2,1 / 2]),[1 / 2,1 / 2]\right)$, and so $[0,1],[1 / 2,1 / 2] \in A$ and $[1,1] \notin A$, where $A=\left\{z \in I\left(L_{3}\right) \mid \mathcal{T}\left(\mathcal{I}_{\mathcal{T}}([0,1],[1 / 2,1 / 2]), z\right) \leq_{t}[1 / 2,1 / 2]\right\}$. Since $[1,1] \notin A$, we have $\mathcal{T}\left(\mathcal{I}_{\mathcal{T}}([0,1],[1 / 2,1 / 2]),[1,1]\right)=$ $\mathcal{I}_{\mathcal{T}}([0,1],[1 / 2,1 / 2]) \quad \mathbb{Z}_{t} \quad[1 / 2,1 / 2], \quad$ hence, $a=\mathcal{I}_{\mathcal{T}}([0,1],[1 / 2,1 / 2]) \in\{[0,1],[1 / 2,1],[1,1]\}$. But $a=[1,1]$ is not possible, since $[0,1] \in A$ and $[0,1] \not_{t}[1 / 2,1 / 2]$. Now, $a \geq_{t}[0,1]$, thus, $\sup B \geq_{t}[0,1]$, where $B=\left\{z \in I\left(L_{3}\right) \mid \mathcal{T}([0,1], z) \leq_{t}[1 / 2,1 / 2]\right\}$. Hence, $[0,1] \in B$. On the other hand, since $\wedge$ is the greatest t-norm on $\left(I\left(L_{3}\right), \leq_{t}\right), \mathcal{T}([0,1],[1 / 2,1 / 2]) \quad \leq_{t}$ $[0,1] \wedge[1 / 2,1 / 2]=[0,1 / 2] \leq_{t}[1 / 2,1 / 2]$, so $[1 / 2,1 / 2] \in B$. Thus, $a=\sup B=[1 / 2,1]$.

Since $\mathcal{T}$ satisfies the residuation principle, from $[1 / 2,1] \leq_{t}$ $a=\mathcal{I}_{\mathcal{T}}([0,1],[1 / 2,1 / 2])$ it follows that $\mathcal{T}([0,1],[1 / 2,1]) \leq_{t}$ $[1 / 2,1 / 2]$. Since $\mathcal{T}([0,1],[1 / 2,1]) \leq_{t}[0,1]$, we obtain that $\mathcal{T}([0,1],[1 / 2,1]) \leq_{t}[0,1 / 2]$.

Since $\mathcal{T}$ satisfies $(8)$, we find that $[1 / 2,1 / 2] \vee[0,1 / 2]=$ $[1 / 2,1 / 2]=\sup C$, where

$$
\begin{aligned}
C=\left\{z \in I\left(L_{3}\right) \mid \mathcal{T}\left(\mathcal{I}_{\mathcal{T}}\left(\left[\frac{1}{2}, \frac{1}{2}\right],\left[0, \frac{1}{2}\right]\right), z\right)\right. & \\
& \left.\leq{ }_{t}\left[0, \frac{1}{2}\right]\right\} .
\end{aligned}
$$

This is only possible if $[1 / 2,1 / 2] \in C$. Furthermore, $[0,1] \notin C$, since otherwise $[0,1] \vee[1 / 2,1 / 2]=[1 / 2,1] \leq_{t} \sup C$, a contradiction. Hence, $\mathcal{T}\left(\mathcal{I}_{\mathcal{T}}([1 / 2,1 / 2],[0,1 / 2]),[0,1]\right) \quad \mathbb{Z}_{t}$ $[0,1 / 2]\}$. Since, from the above we know that $\mathcal{T}([1 / 2,1],[0,1]) \quad \leq_{t} \quad[0,1 / 2], \quad$ it follows that $b=\mathcal{I}_{\mathcal{T}}([1 / 2,1 / 2],[0,1 / 2]) \mathbb{Z}_{t}[1 / 2,1]$, so $b=[1,1]$. By the residuation principle, from $\mathcal{I}_{\mathcal{T}}([1 / 2,1 / 2],[0,1 / 2]) \geq_{t}[1,1]$, we obtain that $\mathcal{T}([1 / 2,1 / 2],[1,1]) \leq_{t}[0,1 / 2]$, which is a contradiction.

We have shown, then, that although there exists a Kleenenegator on $\left(I\left(L_{3}\right), \leq_{t}\right)$, there does not exist a t-norm $\mathcal{T}$ on $\left(I\left(L_{3}\right), \leq_{t}\right)$ such that $\left(I\left(L_{3}\right), \leq_{t}, \mathcal{T}\right)$ is an MV-algebra.

\section{CONCLUSION}

In this paper, we have identified bilattices, and in particular the constructs of bilattice-based squares and triangles, as a natural setting for representing and maintaining contradictions (as we do not restrict ourselves only to the consistent elements). These structures open the door to new opportunities in modeling imprecise information. Indeed, the "traditional" approach of evaluating membership functions by values that are arranged in one (and usually total) order, is replaced here by more expressive "two-dimensional" measurements that reflect different interpretations of the underlying orderings, which may be applied simultaneously.

We have shown that the definition and representation of suitable logical connectives for a given setting can benefit a lot from bringing together results from bilattice theory and $\mathcal{L}$-fuzzy set theory, and - moreover-it raises many nontrivial questions regarding the interrelationships among the various alternative definitions. From the obtained results it should be clear also that the situation in squares and triangles is often substantially different; for instance, neither Kleene negators nor MV-algebras exist on triangles, while for squares these constructs are readily obtained. Yet, both kinds of structures offer, for every basic logical connective, several graded extensions that reflect the semantic nature of our framework as one that supports different forms and levels of uncertainty, vagueness, and inconsistency. This is another vindication to our claim that both squares and triangles are very useful for modeling and representing imprecise information. The choice what structure is more appropriate for practical applications is strongly affected by the nature of the problem under consideration, as well as by representation considerations, such as those given in this paper.

We note, finally, that apart of the intuitive appeal of our framework, it also has an interesting application potential. This is illustrated in a forthcoming paper, where we consider our approach in the context of preference modeling, showing that bilattice-based representation of the underlying problem provides a generic solution strategy that clarifies and simplifies existing works in this area (e.g., [45]-[48]).

\section{REFERENCES}

[1] M. L. Ginsberg, "Multi-valued logics: A uniform approach to reasoning in artificial intelligence," Comput. Intell., vol. 4, pp. 256-316, 1988.

[2] — "Multi-valued logics," in Readings in Non-Monotonic Reasoning. San Mateo, CA: Morgan Kaufmann, 1988, pp. 251-258.

[3] M. Fitting, "Bilattices in logic programming," in Proc. 20th Int. Symp. on Multiple-Valued Logic, G. Epstein, Ed., 1990, pp. 238-246.

[4] — "Bilattices and the semantics of logic programming," J. Logic Program., vol. 11, no. 2, pp. 91-116, 1991.

[5] $\_$, "Kleene's three-valued logics and their children," Fundament. Inf., vol. 20, pp. 113-131, 1994.

[6] B. Mobasher, D. Pigozzi, and G. Slutzki, "Multi-valued logic programming semantics: An algebraic approach," Theoret. Comput. Sci., vol. 171, no. 1-2, pp. 77-109, 1997.

[7] O. Arieli, "Paraconsistent declarative semantics for extended logic programs," Ann. Math. Artif. Intell., vol. 36, no. 4, pp. 381-417, 2002.

[8] Y. Loyer and U. Straccia, J. F. , Ed., "Epistemic foundation of the well-founded semantics over bilattices," in Proc. 29th Int. Symp. Math. Found. Comput. Sci. (MFCS'04), 2004, pp. 513-524.

[9] R. Nelken and N. Francez, "Bilattices and the semantics of natural language questions," Linguist. Philos., vol. 25, no. 1, pp. 37-64, 2002. 
[10] B. Messing, "Combining knowledge with many-valued logics," Data Knowl. Eng., vol. 23, pp. 297-315, 1997.

[11] O. Arieli and A. Avron, "Logical bilattices and inconsistent data," in Proc. 9th Ann. IEEE Symp. Logic in Comput. Sci. (LICS'94), 1994, pp. 468-476.

[12] _ _ , "Reasoning with logical bilattices," J. Logic, Language, Inf., vol. 5, no. 1, pp. 25-63, 1996.

[13] _ , "Bilattices and paraconsistency," in Frontiers of Paraconsistent Logic. Piscataway, NJ: Research Studies, 2000, pp. 11-27.

[14] G. Gargov, "Knowledge, uncertainty and ignorance in logic: Bilattices and beyond," J. Appl. Non-Class. Logics, vol. 9, no. 2-3, pp. 195-283, 1999.

[15] V. B. Tarassov, "Lattice products, bilattices and some extensions of negations, triangular norms and triangular conorms," in Proc. Int. Conf. Fuzzy Sets and Soft Comput. Econom. Finance (FSSCEF'04), I. Batyrshin, J. Kacpzryk, and L. Sherenetov, Eds., 2004, vol. 1, pp. 272-282.

[16] O. Arieli, C. Cornelis, G. Deschrijver, and E. E. Kerre, "Relating intuitionistic fuzzy sets and interval-valued fuzzy sets through bilattices," in Appl. Computat. Intell., D. Ruan, P. D'Hondt, M. De Cock, M. Nachtegael, and E. E. Kerre, Eds., 2004, pp. 57-64.

[17] O. Arieli, C. Cornelis, G. Deschrijver, and E. E. Kerre, "Bilattice-based squares and triangles," Proc. 8th Euro. Conf. Symbol. Quantit. Approaches to Reasoning with Uncertainty (ECSQARU'05) L. Godo, Ed. , ser. Lecture Notes in Artificial Intelligence New York, Springer, 3571, pp. 563-575, 2005.

[18] K. T. Atanassov, Intuitionistic fuzzy sets. Sofia, Bulgaria, 1983, (deposed in Central Sci.-Technical Lib. Bulg. Acad. of Sci., 1697/84) (in Bulgarian). VII ITKR's Session.

[19] R. Sambuc, "Fonctions $\Phi$-floues. Application à l'aide au diagnostic en pathologie thyroidienne," Ph.D. dissertation, Univ. Marseille, Marseille, France, 1975.

[20] I. B. Türkşen, "Interval valued fuzzy sets based on normal forms," Fuzzy Sets Syst., vol. 20, no. 2, pp. 191-210, 1986.

[21] J. Goguen, "L-fuzzy sets," J. Math. Anal. Appl., vol. 18, pp. 145-174, 1967.

[22] M. Fitting, "Kleene's logic, generalized," J. Logic Computat., vol. 1, pp. 797-810, 1992.

[23] C. Cornelis, K. T. Atanassov, and E. E. Kerre, "Intuitionistic fuzzy sets and interval-valued fuzzy sets: a critical comparison," in Proc. 3rd Int. Conf. in Fuzzy Logic and Technol. (EUSFLAT'03), M. Wagenknecht and R. Hampel, Eds., 2003, pp. 159-163.

[24] C. Cornelis, G. Deschrijver, and E. E. Kerre, "Square and triangle: Reflections on two prominent mathematical structures for the representation of imprecision," Notes Intuit. Fuzzy Sets, vol. 9, no. 3, pp. 11-21, 2003.

[25] — - "Implication in intuitionistic and interval-valued fuzzy set theory: Construction, classification, application," Int. J. Approx. Reas., vol. 35 , no. 1 , pp. 55-95, 2004

[26] G. Deschrijver, C. Cornelis, and E. E. Kerre, "On the representation of intuitionistic fuzzy t-norms and t-conorms," IEEE Trans. Fuzzy Syst., vol. 12 , no. 1, pp. 45-61, 2004.

[27] N. D. Belnap, "A useful four-valued logic," in Modern Uses of Multiple-Valued Logic, G. Epstein and J. M. Dunn, Eds. Amsterdam, The Netherlands: Reidel , 1977, pp. 7-37.

[28] — , "How a computer should think," in Contemporary Aspects of Philosophy. Zurich, Switzerland: Oriel, 1977, pp. 30-56.

[29] A. Avron, "A note on the structure of bilattices," J. Math. Struct. Comp. Sci., vol. 5, pp. 431-438, 1995.

[30] _ "The structure of interlaced bilattices," J. Math. Struct. Comp. Sci., vol. 6, pp. 287-299, 1996.

[31] J. M. Font and M. Moussavi, "Note on a six-valued extension of threevalued logic," J. Appl. Non-Class. Logic, vol. 3, no. 2, pp. 173-187, 1993.

[32] M. Kondo, "On the structure of weak interlaced bilattice," in Proc. 32th IEEE Int. Symp. Multiple-Valued Logic (ISMVL'02), 2002, pp. 23-26.

[33] G. Wagner, "Vivid logic," Lecture Notes in Artif. Intell., vol. 764, 1994.

[34] P. Doherty, D. Driankov, and A. Tsoukiàs, Partiality, para-consistency and preference modelling Linköping Univ., IDA Res. Rep. Lith-IDA-R-92-18, 1992.

[35] A. Tsoukiàs, "A first-order, four valued, weakly paraconsistent logic and its relation to rough sets semantics," Found. Comput. Dec. Sci., vol. 27 , pp. 77-96, 2002.
[36] G. Deschrijver, C. Cornelis, and E. E. Kerre, "Square and triangle: A comparison," in Proc. Conf. Inf. Process. Manage. Uncertainty in Knowl-Based Syst. (IPMU'04), 2004, pp. 1389-1396.

[37] O. Arieli and A. Avron, "The value of the four values," Artif. Intell., vol. 102, no. 1, pp. 97-141, 1998.

[38] C. Cornelis, G. Deschrijver, and E. E. Kerre, "Classification of intuitionistic fuzzy implicators: An algebraic approach," in Proc. 6th Joint Conf. Inf. Sci. (JCIS'01), H. J. Caulfield, S. Chen, H. Chen, R. Duro, V. Honavar, E. E. Kerre, M. Lu, M. G. Romay, T. K. Shih, D. Ventura, P. P. Wang, and Y. Yang, Eds., 2002, pp. 105-108.

[39] S. Jenei and B. De Baets, "On the direct decomposability of t-norms on product lattices," Fuzzy Sets Syst., vol. 139, no. 3, pp. 699-707, 2003.

[40] M. De Cock, "A thorough study of linguistic modifiers in fuzzy set theory," Ph.D. dissertation, Ghent Univ., Gent, Belgium, 2002.

[41] B. De Baets and R. Mesiar, "Triangular norms on product lattices," Fuzzy Sets Syst., vol. 104, no. 1, pp. 61-75, 1999.

[42] C. C. Chang, "Algebraic analysis of many valued logics," Trans. AMS93, pp. 74-80, 1958.

[43] E. Turunen, Mathematics Behind Fuzzy Logic. Berlin, Germany: Adv. Soft Comput., Phys.-Verlag, 1999.

[44] C. Cornelis, "Two-sidedness in the representation and processing of imprecise information," Ph.D. dissertation, Ghent Univ., Gent, Belgium, 2004.

[45] A. Tsoukiàs and P. Vincke, "A new axiomatic foundation of partial comparability," Theory Decision, vol. 39, pp. 79-114, 1995.

[46] — - "Extended preference structures in mcda," in Multi-Criteria Analysis. Berlin, Germany: Springer-Verlag, 1997, pp. 37-50.

[47] A. Tsoukiàs and P. Perny, , P. Vincke, D. Bouyssou, E. Jacquet-Lagrèze, P. Perny, R. Slowiński, and D. Vanderpooten, Eds., "From concordance/discordance to the modelling of positive and negative reasons in decision aiding," in Aiding Decisions with Multiple Criteria: Essays in Honor of Bernard Roy. Dordrecht, The Netherlands: Kluwer, 2002, pp. 147-174.

[48] P. Fortemps and R. Słowiński, "A graded quadrivalent logic for ordinal preference modelling: Loyola-like approach," Fuzzy Optimiz. Decision Making, vol. 1, pp. 93-111, 2002.

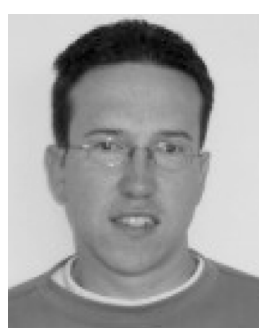

Chris Cornelis received the Ph.D. degree in computer science in 2004 from Ghent University, Gent, Belgium, with a thesis on "two-sidedness in the representation and processing of imprecise information."

At present, he is a Postdoctoral Fellow with the Fuzziness and Uncertainty Modelling Research Unit, Department of Applied Mathematics and Computer Science, Ghent University. His research interests include various models of imprecision (fuzzy rough sets, bilattices, interval-valued and intuitionistic fuzzy sets, $\mathcal{L}$-fuzzy sets), data mining, approximate reasoning. He is currently focusing on their application to personalized information access and web intelligence.

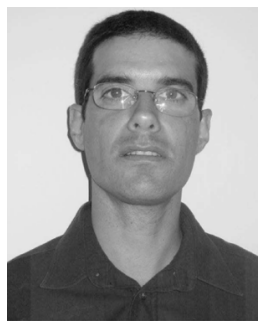

Ofer Arieli received the B.Sc. degree in mathematics and computer science from the Hebrew University of Jerusalem in 1987, and the M.Sc. and Ph.D. degrees in computer science from Tel-Aviv University, Israel, in 1992 and 1999, respectively.

$\mathrm{He}$ is a Senior Lecturer with the Department of Computer Science, Academic College of Tel-Aviv. His research interests are related to applications of logic in artificial intelligence, in particular, nonmonotonic logics for reasoning with incomplete and inconsistent information, knowledge representation, 


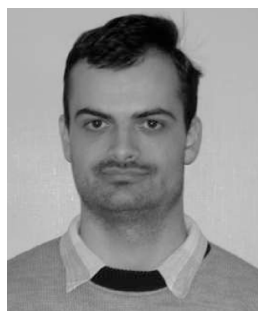

Glad Deschrijver received the M.Sc. degree in mathematics from Ghent University, Gent, Belgium, in 1999. He received the "Qualified Teacher's Degree for Higher Secondary Education-Section 2 in mathematics." He received the Ph.D. degree in 2004 in science and mathematics, with a thesis on intuitionistic fuzzy sets

In summer 2000, he joined the Department of Applied Mathematics and Computer Science, Ghent University, where he is a member of the Fuzziness and Uncertainty Modelling Research Unit. His research interests include the mathematics of different models of imprecision, including interval-valued fuzzy sets, intuitionistic fuzzy sets in the sense of Atanassov, $\mathcal{L}$-fuzzy sets and bilattices. He is currently doing research on triangular norms and related operators in interval-valued fuzzy set theory.

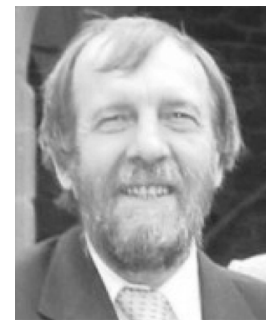

Etienne E. Kerre received the M.Sc. degree in mathematics in 1967 and the Ph.D. degree in mathematics in 1970 from Ghent University, Gent, Belgium.

Since 1984, he has been a lector, and since 1991, a full professor with Ghent University. He is a referee for more than 50 international scientific journals. In 1976, he founded the Fuzziness and Uncertainty Modelling Research Unit (FUM), Ghent University, and since then his research has been focused on the modeling of fuzziness and uncertainty, and has resulted in a great number of contributions in fuzzy set theory and its various generalizations. The theories of fuzzy relational calculus and fuzzy mathematical structures are owed a great deal to him. Over the years, he has also been a supervisor of $20 \mathrm{Ph} . \mathrm{D}$. students on fuzzy set theory. His current research interests include fuzzy and intuitionistic fuzzy relations, fuzzy topology, and fuzzy image processing. He has authored or coauthored 11 books, and more than 300 papers appeared in international refereed journals and proceedings.

Dr. Kerre is a member of the editorial board of international journals and conferences on fuzzy set theory. He was an honorary chairman at various international conferences. 